$$
\begin{aligned}
& \text { APPENDIX } 5 \\
& \text { Submithd to the AIAA JOUPNAL }
\end{aligned}
$$

\title{
EQUIVALENT SKIN ANALYSIS OF WING STRUCTURES USING
}

\section{NEURAL NETWORKS}

\author{
Youhua Liu* and Rakesh K. Kapania** \\ Virginia Polytechnic Institute and State University \\ Blacksburg, VA 24061
}

\begin{abstract}
An efficient method of modeling trapezoidal built-up wing structures is developed by coupling. in an indirect way, an Equivalent Plate Analysis (EPA) with Neural Networks (NN). Being assumed to behave like a Mindlin-plate, the wing is solved using the Ritz method with Legendre polynomials employed as the trial functions. This analysis method can be made more efficient by avoiding most of the computational effort spent on calculating contributions to the stiffness and mass matrices from each spar and rib. This is accomplished by replacing the wing inner-structure with an "equivalent" material that combines to the skin and whose properties are simulated by neural networks. The constitutive matrix, which relates the stress vector to the strain vector, and the density of the equivalent material are obtained by enforcing mass and stiffness matrix equities with regard to the EPA in a least-square sense. Neural networks for the material properties are trained in terms of the design variables of the wing structure. Examples show that the present method, which can be called an Equivalent Skin Analysis (ESA) of the wing structure, is more efficient than the EPA and still fairly good results can be obtained. The present ESA is very promising to be used at the early stages of wing structure design.
\end{abstract}

*: Research Assistant, Department of Aerospace and Ocean Engineering. Member AIAA **: Professor. Department of Aerospace and Ocean Engineering. Associate Fellow AIAA 


\section{Introduction}

Traditionally, the conceptual design is often carried out using simplified relations that have been learned from previous similar designs. This approach can only result in incremental advancements in technology because experience-based design makes large step design extrapolations too risky. This risk can be reduced significantly if the design is based on physicsbased high-fidelity models since using these models one can predict the consequences of large design extrapolations with a greater confidence.

Physics-based high-fidelity models, such as those in the Finite Element Analysis (FEA) for structures, Computational Fluid Dynamics (CFD) for aerodynamic loads etc., are increasingly being used as early as possible in airplane design. This is not surprising because it is estimated that about $90 \%$ of the cost of a product is committed during the first $10 \%$ of the design cycle, and an accurate analysis is often crucial to obtaining a good estimate of the manufacturing and life-cycle costs of a product.

For structural analysis, the Finite Element Method (FEM) is widely used because of its generality, versatility and reliability, but its use in the early design stages faces some major obstacles: A prohibitive preparation time for the FE model data, and a large amount of CPU time for problems with a high number of degrees of freedom. This is especially true for complex builtup structures such as the airplane wings.

In view of this situation, Kapania and Liu $^{1}$ have developed an efficient method, using the equivalent plate analysis (EPA), for studying the static and vibration problems of general trapezoidal built-up wing structures composed of skins, spars, and ribs. The model includes the transverse shear effects by treating the built-up wing as a plate following the Reissner-Mindlin theory (FSDT). The Ritz method is used with the Legendre polynomials being employed as the trial 
functions. The Legendre polynomials have the orthogonality property, and this is an advantage over using simple-polynomials as the trial functions, which is known to be prone to numerical illconditioning problems.

But there is a problem in the method in Kapania and Liu' ', that is, since complex trial functions are used, the stiffness and mass matrices cannot be integrated analytically, as in the case of Livne ? and numerical integration over every item of the structural components takes a large part of the computing effort. For instance, in solving a free vibration problem, evaluating various matrices requires about $2 / 3$ of the total CPU time.

This problem is addressed in this paper. Instead of evaluating the matrices over all components of the wing structure, evaluation is performed only over the skins, whose "equivalent" material constitutive matrix and mass density distribution are changed accordingly to incorporate the spar and rib effects. The new skin material properties are simulated using Neural Networks in terms of the wing design variables. As shall be shown, while the Neural-Network-aided EPA, which will be called Equivalent Skin Analysis (ESA) hereafter, gives almost equally good results, it uses only a fraction of the CPU time spent in the ordinary EPA in evaluating the matrices.

\section{An Equivalent Plate Analysis (EPA) Method}

The Reissner-Mindlin method, a First-order Shear Deformation Theory (FSDT), is based on two assumptions for the displacement field: (1) A straight line normal to the non-deformed middle surface remains a straight, but not necessarily normal to the deformed surface, line after deformation: and (2) The transverse normal stress can be neglected in the constitutive relations.

We want to analyze a trapezoidal wing by assuming that it behaves as a plate whose deformation satisfies the Reissner-Mindlin displacement field. For the convenience of calculation. a 
coordinate transformation from $(x, y)$ to $(\xi, \eta)$ is performed, which transforms the wing plan surface from the original trapezoidal to a square. Details of the coordinate transformation are available in Ref. 1.

By representing the displacement components as the sum of terms $P_{i-1}(\xi) P_{j-1}(\eta)$, where $P$ is are the Legendre polynomials, $i=1, \cdots, I, j=1, \cdots, J$ ( $I, J$ are integers), we can call the vector composed of all the coefficients of the terms as the generalized displacement vector. Based on this displacement vector and considering the total strain and kinetic energy of the wing structure, we can derive the stiffness and mass matrices as in the following forms:

$$
\begin{aligned}
& {[K]=\iiint_{V}[C]^{T}[T]^{T}[D][T][C] d V} \\
& {[M]=\iiint_{V} \rho[H]^{T}[Z Z][H] d V}
\end{aligned}
$$

where the integration domain $V$ includes all and only the spaces which the components of the wing occupy. Details of obtaining matrix $[C]$ etc. can be found in Ref. 1. For different wing components, skin, spar or rib, the material constitutive matrix should be treated differently'. The integration in the $(\xi, \eta)$ plane is performed using the Gaussian quadrature.

The boundary conditions are approximated by applying springs with very large magnitudes of stiffness on the boundaries. For clamped edges both linear and rotational springs are needed, and for simply supported edges only linear springs are used. An integration on the boundaries gives $\left[K_{\text {,prng }}\right]$. The total stiffness and mass matrices of the wing structure is given as

$$
\begin{aligned}
& {\left[K_{\text {sural }}\right]=\left[K_{\text {struin }}\right]+\left[K_{\text {spring }}\right],\left[K_{\text {strain }}\right]=\left[K_{\text {skin }}\right]+\left[K_{\text {spar }}\right]+\left[K_{r b}\right],} \\
& {\left[M_{\text {sortal }}\right]=\left[M_{s k \text { in }}\right]+\left[M_{\text {spar }}\right]+\left[M_{r i b}\right] .}
\end{aligned}
$$


The natural frequencies and mode shapes for the free vibrating wing can be determined by applying the Lagrange's equations, which results in the following eigenvalue problem

$$
\left[K_{\text {trotal }}-\lambda M_{\text {tortal }}\right](x)=0
$$

where $\lambda=\omega^{2}$ is an eigenvalue of the system of equations, $\omega$ is the corresponding frequency in radians/second, and $\{x\}$ is the corresponding eigenvector.

Static problems (including stress distributions) can be readily solved by making use of the total stiffness matrix $\left[K_{\text {totul }}\right]$. For details one can refer to Ref. 1.

Compared with the methods in Kapania and Lovejoy ${ }^{3}$ and Cortial ${ }^{4}$, the formulation in Ref. 1 is such that there is no limitation on the wing thickness distribution. As shown in Ref. 1, the method shows a good performance for both static and vibration problems in comparison with the FEA using MSC/NASTRAN.

As Eqs. (1) and (2) are being evaluated by numerical schemes such as the Gauss quadrature ', the major computing effort is spent in calculating the contributions from the large number of the local components, namely, spars and ribs. The purpose of the present paper is to reduce the computing effort in obtaining $[K]$ and $[M]$ by incorporating the contributions from those local components to the global components such as the skin. This is accomplished by postulating a new material constitutive matrix and mass distribution for the skins and relating the terms of the constitutive matrix and mass density to various design variables of the wing structure by using Neural Networks, as shall be shown in the following sections. Design variables for wing structure can be sizing-type variables (skin thickness, spar or rib sectional area etc.), shape variables (the plan surface dimensions and ratios), and topological variables (total spar or rib number, wing topology arrangements etc.). 


\section{Application of Neural Networks in Structural Problems}

Artificial Neural Networks (ANN), or simply Neural Networks (NN) are computational systems inspired by the biological brain in their structure, data processing and restoring method, and learning ability. More specifically, a neural network is defined as a massively parallel distributed processor that has a natural propensity for storing experiential knowledge and making it available for future use by resembling the brain in two aspects: (i) Knowledge is acquired by the network through a learning process; (ii) Inter-neuron connection strengths known as synaptic weights (or simply weights) are used to store the knowledge ${ }^{5}$.

Major steps of utilizing $\mathrm{NN}$ include: (i) specifying the topology or the structural parameters (number of layers, number of neurons in each layer, etc.) of the $\mathrm{NN}$, (ii) training of the $\mathrm{NN}$, corresponding to the learning process of the brain, (iii) simulation, corresponding to the recalling function of the brain.

The NN has the following properties: (i) Many of its kind are universal approximators, in the sense that, given a dimension (number of hidden layers and neurons of each layer) large enough, any continuous mapping can be realized; Therefore, (ii) a NN provides a general mechanism for building models from data, or gives a general means to set up input-output mapping (iii) The input and output relationship of NN can be highly nonlinear; (iv) A NN is parallel in nature, and it can make computation fast when executed in a parallel computer, though $\mathrm{NN}$ can be simulated in ordinary computers in a sequential manner.

Neural Network has found numerous applications in science and engineering, from biological and medical sciences, to information technologies such as artificial intelligence, pattern recognition, signal processing and control, and to engineering areas as civil and structural engineering. In the field of structural engineering, there have been a lot of attempts and researches 
making use of $\mathrm{NN}$ to improve efficiency or to capture relations in complex analysis or design problems. The following are a few examples. Hajela and Berke ${ }^{6}$ gave an overview of the neural computing approach, and examined the role of neural computing strategies in structural analysis and design. Abdalla and Stavroulakis ${ }^{7}$ applied $\mathrm{NN}$ to represent experimental data to model the behavior of semi-rigid steel structure connections, which are related to some highly nonlinear effects such as local plastification etc. Several cases of neural network application in structural engineering can be found in Vanluchene and Sun ${ }^{8}$. All the problems treated in Ref. 8 had been reproduced in Gunaratnam and Gero ${ }^{9}$ with a conclusion that representational change of a problem based on dimensional analysis and domain knowledge can improve the performance of the networks. In Liu, Kapania and VanLandingham ${ }^{10}$, methodologies of applying Neural Networks and Genetic Algorithms to simulate and synthesize substructures were explored in the solution of 1 $\mathrm{D}$ and 2-D beam problems.

For the efficient simulation of the structural performances of complex wings, there can be two directions to apply NN as specified in the following:

\section{(1) Direct Application}

In this case, the input layer includes all the design variables of interest (for instance, the four shape parameters of the wing plan form). The output layer gives the desired structural responses, such as natural frequencies etc. The EPA is being used as the training data generator. though if necessary, results obtained using the FEM can also be used as the training data. Preparation of training data is very important, and the training algorithm used also greatly impacts the process of training ${ }^{\prime}$. Caution must be taken in specifying the network parameters and training criterion, such that the results of the trained network would not oscillate around the 
training data. Once the networks are trained, structural responses at any design point can be recalled in a fraction of a second and this is really favorable in a design situation ${ }^{12}$.

\section{(2) Indirect Application}

Here it is desired to find a way of incorporating $\mathrm{NN}$ into the application of the equivalent plate analysis (EPA) of complex wing structures, other than just making use of results generated by EPA as the training data base. Note that in the EPA of a complex wing, the computation effort is mainly spent on integrals for generating the contribution from the inner-structural components of the wing, the spars and the ribs, to the stiffness and mass matrices. If an equivalent anisotropic material can be found to replace the inner components, in terms of an equivalent skin, such that the new composite wing has similar global properties as the original one, then the EPA can be performed more efficiently. Solution of the adequate material properties of the anisotropic material is the major obstacle here. The role of $\mathrm{NN}$ will be relating the material properties to all kinds of wing design parameters, when there exists enough data base for training. This way of applying NN has been claimed to be the best use of the Neural Networks in structural engineering ${ }^{9}$ :

"The real usefulness of neural networks in structural engineering is not in reproducing existing algorithmic approaches for predicting structural responses, as a computationally efficient alternative, but in providing concise relationships that capture previous design and analysis experiences that are useful for making design decisions."

This is the path that we followed in this study. 


\section{Equivalent Skin Analysis (ESA) of Wing Structures}

Since the calculation of various integrals in Eqs. (1) and (2) are time-consuming, it is desired to replace the actual wing structure by an equivalent continuum model, that is, one that is composed of a skin-like material, whose constitutive matrix $[D]$ and distribution of mass in Eqs. (1) and (2) are to be decided.

It is assumed that the mass density $\rho$ is a function of position in the plan form while each term of $[D]$ is a constant throughout the wing area. There can be other choices, as will be discussed later.

We are going to solve the above problem by requiring that the stiffness and mass matrices of the equivalent model are most approximate to those of the actual wing in a least squares sense. This gives the following procedures.

\section{(a) The Constitutive matrix}

Let's write $[K]=\left[K_{\text {strain }}\right]=\left[K_{i j}\right]$ as the target matrix, and the stiffness matrix of the equivalent continuum movel is

$$
\begin{aligned}
& {[\tilde{K}]=\sum_{m} \sum_{n} g_{m} g_{n}[C]_{m n}^{T}[T]_{m n}^{T}[D][T]_{m n}[C]_{m n}} \\
& =\left[\tilde{K}_{i}\right]=\left[\sum_{p} \sum_{q} D_{p q}\left(\sum_{m} \sum_{n} \bar{G}_{p q, m n}^{i j}\right)\right]=\sum_{p, q} D_{p q}\left[G_{p q}^{i j}\right]
\end{aligned}
$$

where $g_{m}$ and $g_{n}$ are the Gauss quadrature weights; the constitutive matrix $[D]$ relates the stress and strain vectors by $\{\sigma\}=[D]\{\varepsilon\}$, and $D_{p q}$ is the $p$-th row, $q$-th column term of the constitutive matrix;

$m(m=1, \cdots, K)$ corresponds to the $m$-th Gauss integration position in the $\mathrm{x}$-direction.

$K$ is an integer with a usual value of 6 or 8 ;

$$
n(n=1, \cdots, K) \text { corresponds to the } n \text {-th Gauss integration position in the } y \text {-direction; }
$$




$$
\begin{aligned}
& p=1, \cdots, 5 \text { is the row number of }[D] \\
& q=1, \cdots, 5 \text { is the column number of }[D]
\end{aligned}
$$

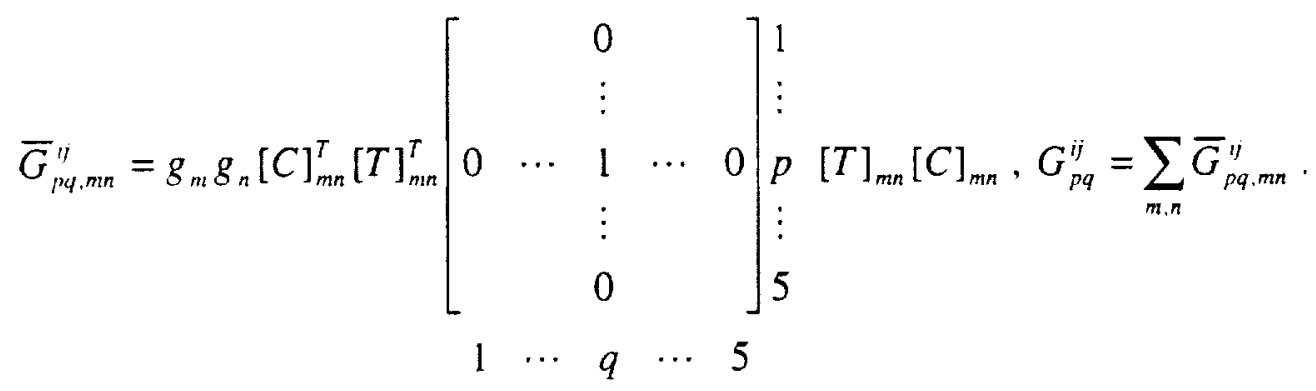

By constructing an error function

$$
E_{K}=\sum_{i, j} w_{i j}^{K}\left[\tilde{K}_{i j}\left(D_{p q}\right)-K_{i j}\right]^{p}
$$

where $w_{i j}^{K}$ are the weight coefficients, and by requiring

$$
\frac{\partial E_{K}}{\partial D_{p q}}=2 \sum_{i, j} w_{i j}^{k}\left[\tilde{K}_{i j}\left(D_{p q}\right)-K_{i j}\right] \frac{\partial \tilde{K}_{i j}}{\partial D_{p q}}=0
$$

and noting

$$
\frac{\partial \tilde{K}_{i j}}{\partial D_{p q}}=G_{p q}^{i j}\left(G_{p q}^{i j} \text { means the } i, j \text {-th term of matrix }\left[G_{p q}\right]\right)
$$

we can obtain the constitutive matrix term $\left[D_{p q}\right]$ by solving the following linear equation set:

$$
\begin{aligned}
& \quad \sum_{p^{\prime} \cdot q^{\prime}}\left(\sum_{i, j} w_{i, j}^{K} G_{p, q^{i j}}^{i j} G_{p q}^{i j}\right) D_{p^{\prime} q^{\prime}}=\sum_{i, j} w_{i j}^{K} K_{i j} G_{p q}^{i j} \\
& i, j=1, \cdots, K \text { and } p, q, p^{\prime}, q^{\prime}=1, \cdots, 5 .
\end{aligned}
$$

This is an equation set with 25 unknowns. Since $i, j=1, \cdots, N$ and $N=5 K^{2}$ is usually very large (if we use the Legendre polynomials of 6 terms as the basis functions, $N=180$, if 8 terms are used, then $N=320$ ), the job of generating the matrix in Eq. (9) will be very extensive. 
If $[D]$ is assumed to be symmetrical, then Eq. (9) will become

$$
\sum_{i p^{\prime}, q^{\prime}}\left(\sum_{i, j} w_{i j}^{K}\left\{G_{p q^{\prime}}^{i j}+\left(1-\delta_{p q^{\prime}}\right) G_{q^{\prime} p^{i j}}^{j}\right\} G_{p q}^{i j}\right) D_{p^{\prime} q^{\prime}}=\sum_{i, j} w_{i j}^{K} K_{i j} G_{p q}^{i j}
$$

where $\left(p^{\prime}, q^{\prime}\right)$ and $(p, q)$ have the following 15 instead of 25 combinations: $(1,1),(1,2),(1,3)$, $(1,4),(1,5),(2,2),(2,3),(2,4),(2,5),(3,3),(3,4),(3,5),(4,4),(4,5)$, and $(5,5)$.

\section{(b) Mass distribution}

Let's write $[M]=\left[M_{t u r a l}\right]=\left[M_{t \prime}\right]$ as the target matrix, and

$$
[\tilde{M}]=\sum_{m} \sum_{n} \rho_{m, n} g_{m} g_{n}[H]_{m n}^{T}[Z]_{m n}^{T}[Z]_{m n}[H]_{m n}=\sum_{m} \sum_{n} \rho_{m n}[F]_{m n}
$$

as the mass matrix of the continuum model, where $m(m=1, \cdots, K)$ corresponds to the $m$-th Gauss integration position in the $\mathrm{x}$-direction, $n(n=1, \cdots, K)$ corresponds to the $\mathrm{n}$-th Gauss integration position in the y-direction, $\rho_{m n}$ is the mass density of the equivalent model at position $(m, n), g_{m}$ and $g_{n}$ are integration weights, and $[F]_{m n}=[H]_{m n}^{T}[Z]_{m n}^{T}[Z]_{m n}[H]_{m n}$ is a $N \times N$ matrix varying with position $(m, n)$.

By constructing an error function

$$
E_{M}=\sum_{1.1} w_{i j}{ }^{M}\left[\tilde{M}_{y j}\left(\rho_{m n}\right)-M_{1 j}\right]^{2}
$$

where $w_{t j}^{H}$ are weight coefficients, and by requiring

$$
\frac{\partial E_{M}}{\partial \rho_{m n}}=2 \sum_{i, j} w_{i j}^{M}\left[\tilde{M}_{i j}\left(\rho_{m n}\right)-M_{i j}\right] \frac{\partial \tilde{M}_{i j}}{\partial \rho_{m n}}=0
$$

and noting

$$
\frac{\partial \tilde{M}_{i j}}{\partial \rho_{m n}}=F_{m n}^{i j} \quad\left(F_{m n}^{i j} \text { is the } i, j \text {-th term of matrix } F_{m n}\right),
$$


we can obtain the mass distribution $\rho_{m n}$ by solving the following linear equation set with $K^{\text {? }}$ unknowns:

$$
\sum_{m, n}\left(\sum_{i, j} w_{1 !}^{\prime \prime} F_{m n}^{\prime \prime} F_{m m}^{i j}\right) \rho_{m \cdot n}=\sum_{i, j} w_{i j}^{n} M_{i j} F_{m n}^{i j}, m, n=1, \cdots, K, m^{\prime}, n^{\prime}=1, \cdots, K .
$$

In the present study the following weight coefficients are used:

$$
\left.\begin{array}{l}
w_{i j}^{K}=10\left|K_{i j}^{S k i n}\right| / \max _{i . j}\left(\left|K_{i j}^{S k i n}\right|\right)+1 \\
w_{i j}^{H}=10\left|M_{i j}^{S k i n}\right| / \max _{i, j}\left(\left|M_{i j}^{S k k n}\right|\right)+1
\end{array}\right\}
$$

The basic idea behind this choice is that we want to form the equivalent matrices more in the way of the skin's, which is more like a plate than the other components of the wing, i.e. spars and ribs.

Several choices about the variation of $\rho$ and $[D]$ have been tried, but it is found that the present assumptions give the best results in terms of feasibility and accuracy. For instance, to be consistent with the assumption that each term of $[D]$ is a constant throughout the wing area, $\rho$ can also be assumed a constant. This certainly decreases the accuracy of the method due to the loss of flexibility in varying $\rho$ to simulate the target mass matrix $[M]$, but the resultant reduction in computational effort is small since in the first place, forming Eq. (13) and training the $\rho$-related neural networks do not need much CPU time. In other cases, $[D]$ was assumed to be variable in the span-wise direction or throughout the wing area, but it is found that although the equivalent material is more flexible to simulate the target stiffness matrix $[K]$, the resultant $[\tilde{K}]$ usually has a larger abstract error and the solution of the free vibration problem usually gives worse natural frequencies. Moreover, the CPU time needed for generating Eq. (10), which requires the major computational effort in our method, increases in a factor of about $K$ (number of Gauss integration points, usually with a value of 6 ) in the case of $[D]$ being variable in the span-wise direction. In the 
case of $[D]$ being variable throughout the wing area, the increase can be as large as $K^{2}$ times. As we shall see in the following examples, these kinds of increase in CPU time are formidable.

\section{Examples and Discussion}

The Neural-Network-aided equivalent plate analysis, or briefly Equivalent Skin Analysis (ESA) method is compared with the ordinary equivalent plate analysis (EPA) ${ }^{1}$ in three cases where 4 to 6 design variables are involved respectively. In some of the results, FEA results employing MSC/NASTRAN are also provided as a benchmark.

Some common parameters of the built-up wing structures will be specified herein if not specified otherwise in the following cases. The sections were generated by the Karman-Trefftz transformation $^{13}$. The section thickness-chord ratio varies from 0.15 at the root to 0.06 at the tip. Skin Thickness $t_{0}=.118 \mathrm{in} ;$ Spar Cap Height $h_{1}=.197 \mathrm{in}$, Spar Cap Width $l_{1}=.373 \mathrm{in}$, Spar Web Thickness $t_{1}=.058 \mathrm{in}$ (for definition of $h_{1}$ etc. one can see Ref. 1 ); the ribs have the same cap dimensions and web thickness as the spars. Mass Density $\rho=2.526 \times 10^{-4} l b \cdot \mathrm{sec}^{2} / \mathrm{in}^{4}$, Young's Modulus $E=1.025 \times 10^{7} \mathrm{lb} / \mathrm{in}^{2}$, Poisson's Ratio $v=0.3$. The wing is clamped at the root.

\section{(a) Four-variable case: design space I}

In this case 4 spars and 10 ribs are evenly distributed inside the wing plan form under the skins. For a trapezoidal wing, there are four major independent shape variables: sweep angle $\Lambda$, aspect ratio $\alpha$, taper ratio $\imath$, and plan area $A$ (see Fig. 1). A $3^{4}$ full factorial experimental design with 3 levels in $\Lambda, \alpha, \imath$ and $A$ respectively, was used. Particulars of the levels of every variable are as follows:

$$
\left.\begin{array}{l}
\Lambda=\left\{0^{3}, 15^{3}, 30^{3}\right\}, \quad \alpha=\{1.0,1.75,2.5\}, \\
\tau=\{0.3,0.45,0.6\}, \quad A=\{2000,3500,5000\} \mathrm{in}^{2} .
\end{array}\right\}
$$


For each point in this design space, the EPA is carried out, then Eqs. (10) and (13) are used to generate the 15 constitutive matrix terms and mass densities at $36(6 \times 6)$ Gauss sampling points. and the ESA is performed. For each of these parameters, a feed-forward neural network with a structure of $4 \times 15 \times 10 \times 1$, i.e. 4 inputs, 15 neurons in the first hidden layer, 10 neurons in the second hidden layer, and 1 output, is trained using the MATLAB NN Toolbox function trainlm that trains feed-forward network with the Levenberg-Marquardt algorithm ${ }^{10}$. Therefore, there are totally $15+36=51$ networks to be trained. There are $81\left(3^{+}\right)$sets of training data, which are nondimensionalized before the training process. Once the networks are trained, the input-output relationships can be readily retrieved by using the MATLAB function simuff.

The major computational effort was spent in generating the 81 sets of training data, with about 45 hours of CPU time being spent on a PIV350 personal computer, while less than 1 hour of CPU time being used in training the neural networks. A set of results are given in Fig. 2 where 25 points, which mean 25 new designs, were randomly chosen within the design space box. Upon each new design both the EPA and the ESA are performed. The plan forms of the new design are shown in Fig. 2(a). The first 10 natural frequencies by the EPA and the ESA are compared in Fig. 2(b) and their relative differences (based on the EPA results) are shown in Fig. 2(c). It can be seen that except for a very few cases ( 3 out of 250 ), the relative difference is within $-10 \% \sim 10 \%$.

Fig. 3(a) shows 16 new designs through a randomly chosen path inside the design space box which is defined as

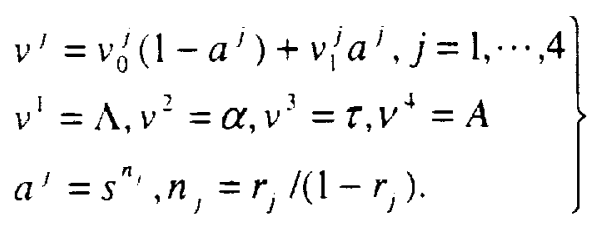


where $v_{0}^{\prime}$ and $v_{1}^{\prime}$ are the lower and upper bounds of variable $v^{\prime}$, for instance, $v_{0}^{\prime}=0^{\circ}, v_{1}^{\prime}=30^{\prime}$

etc. $s \in[0.1]$ is the range of a shape variable, and $r_{j}(j=1, \cdots 4)$ are randomly determined values between 0 and 1 . Results of natural frequencies of the first 6 modes for wing structures defined by points along a path with $n_{1}=0.945, n_{2}=8.200, n_{3}=3.203$ and $n_{4}=1.778$ are shown in Fig. 3 (b), where it can be seen that results by the EPA and the ESA agree with each other quite well.

While Figs. 2 and 3 are about free vibration frequencies, Fig. (4) shows some static results. For an arbitrary new design whose plan form as shown in Fig. 7(a), a down-ward (-z direction) point force of $1 \mathrm{lb}$ is applied at the mid-point of the wing tip (actually the force is divided into components acting on the two spar tips close to the mid-point). Fig. 4(a) shows displacements along the leading-edge by the EPA and the ESA, where $u, v, w$ are displacement components in the chord-wise, span-wise, and vertical directions respectively. Figs. 4(b) and 4(c) show the Von Mises stress distributions at the wing root and the central span-wise line respectively. It can also be seen that the EPA and the ESA give very comparable results for the static case.

\section{(b) Six-variable case: design space II}

In this case spars and ribs are evenly distributed inside the wing plan form but their numbers are design variables. A $3^{6}$ full factorial experimental design with 3 levels in $\Lambda, \alpha, \tau, A$ and numbers of spars and ribs, $n_{\text {spar }}$ and $n_{r i b}$ respectively, was used. Particulars of the levels of every variable are as follows:

$$
\left.\begin{array}{cc}
\Lambda=\left\{0^{\circ}, 15^{\circ}, 30^{\circ}\right\}, & \alpha=\{1 \cdot 0,1 \cdot 75,2.5\}, \\
\tau=\{0.3,0.45,0.6\}, & A=\{2000,3500,5000\} \mathrm{in}^{2}, \\
n_{\text {spar }}=\{2,4,6\}, & n_{r i b}=\{7,10,13\} .
\end{array}\right\}
$$

Similar to case I, for each point in the design space II, the EPA is carried out and Eqs. (10) and (13) are used to generate the 15 constitutive matrix terms and the 36 mass densities which are then 
used to perform the ESA. A total of 51 feed-forward neural networks with a structure of $6 \times 15 \times 10 \times 1$ are trained using the MATLAB NN Toolbox function trainlm. There are $729\left(3^{\circ}\right)$ sets of data that could be used for training, but it was found that at some design points the differences between the natural frequencies by the EPA and the ESA become too large. Therefore, a screening process was introduced, in which any point where the maximum relative difference between the first 10 natural frequencies by the EPA and the ESA surpasses $20 \%$ will be discarded. This led to the removal of 28 points through the process, therefore 701 sets of data were used for training.

Generating the 729 sets of pre-training data used about 152 hours of CPU time on the Crunch (SGI Origin 2000 with eight R10000 processors) of the College of Engineering, Virginia Tech, and training the neural networks spent about 2 hours on a PII/350 PC. A set of results are given in Fig. 5 where 25 points were randomly chosen within the design space box. The plan forms of the new designs are shown in Fig. 5(a), where dashed lines indicate the spar or rib positions. The first 10 natural frequencies by the EPA and the ESA are compared in Fig. 5(b) and their relative differences (based on the EPA results) are shown in Fig. 5(c). It can be seen that the relative difference is within $-5 \% \sim 15 \%$.

Fig. 6(a) shows 16 new designs through a randomly chosen path inside the design space box which is defined as

$$
\left.\begin{array}{l}
v^{\prime}=v_{0}^{j}\left(1-a^{\prime}\right)+v_{1}^{j} a^{j}, j=1, \cdots, 6 \\
v^{\prime}=\Lambda, v^{2}=\alpha, v^{3}=\tau, v^{+}=A, v^{5}=n_{\text {ipar }}, v^{6}=n_{r i b} \\
a^{j}=s^{n,}, n_{j}=r_{j} /\left(1-r_{j}\right) .
\end{array}\right\}
$$

where $r,(j=1, \cdots, 6)$ are randomly determined values between 0 and 1 , and see Eq. (15) for the definition of other symbols. Results of natural frequencies of the first 6 modes for wing structures defined by points along a path with $n_{1}=0.2243, n_{2}=0.8591, n_{3}=0.2064, n_{4}=3.0700, n_{5}=2.2196$ 
and $n_{6}=0.9440$ are shown in Fig. 6(b), where it can be seen that results by the EPA and the ESA agree with each other quite well.

Now some static results. For an arbitrary new design whose plan form is shown in Fig. 7(a), a down-ward (- $z$ direction) point force of $1 \mathrm{lb}$ is applied at the mid-point of the wing tip. Fig. 7(b) shows displacement components along the leading-edge by the EPA and the ESA, compared FEA using MSC/NASTRAN. Figs. 7(c) and 7(d) show the Von Mises stress distributions at the wing root and the central span-wise line respectively together with the FEA results. Comparison of the natural frequencies of this wing as given by the EPA, the ESA and the FEA is shown in Table 1 . It can be seen that the EPA and the ESA results are close, and they all agree quite well with the FEA results.

\section{(c) Design space III}

In this case a wing plan with $\Lambda=30^{\circ}, s=192 \mathrm{in}, b=72 \mathrm{in}$, and $a=36 \mathrm{in}$ (see Fig. 1 for definitions of $s, b$, and $a$ ) is used. A $2^{4} \times 3^{2}$ full factorial experimental design with 2 levels in $t_{0 t}$ (skin thickness at wing tip), $a_{n}$ (skin thickness increment ratio at root over the tip), $h_{1}$ (spar cap height) and $h_{2}$ (rib cap height), and 3 levels in $n_{r p a r}$ and $n_{r b}$, is carried out. The skins are assumed to vary linearly from the root to the tip. Particulars of design space III are as follows:

$$
\left.\begin{array}{cc}
t_{0 t}=\{1,3\} \times 0.118 \mathrm{in}, & a_{\pi}=\{0,2\}, \\
h_{1}=\{1,3\} \times 0.197 \mathrm{in}, & h_{2}=\{1,3\} \times 0.197 \mathrm{in}, \\
n_{\text {spar }}=\{2,4,6\}, & n_{r b b}=\{6,10,14\} .
\end{array}\right\}
$$

There are 144 sets of data for training. Generating these data sets used much less CPU time than in the case of design space II (about 30 hours). A set of results are given in Fig. 8 where 16 points were randomly chosen within the design space box. The plan forms of the new designs are shown in Fig. 8(a), where dashed lines indicate the spar or rib positions, and the skin thickness at the wing 
root and tip. and cap heights of spars and ribs are represented as shown in Fig. 10(a). The first 10 natural frequencies by the EPA and the ESA are compared in Fig. 8(b) and their relative differences (based on the EPA results) are shown in Fig. 8(c). It can be seen that the relative difference is within $-5 \%-15 \%$

Fig. 9(a) shows 16 new designs through a randomly chosen path inside the design space box which is defined as

$$
\left.\begin{array}{l}
v^{\prime}=v_{0}^{\prime}\left(1-a^{j}\right)+v_{1}^{j} a^{j}, j=1, \cdots, 6 \\
v^{\prime}=t_{0 t}, v^{2}=a_{\pi}, v^{3}=h_{1}, v^{+}=h_{2}, v^{5}=n_{s p a r}, v^{6}=n_{r b} \\
a^{\prime}=s^{n_{j}}, n_{j}=r_{j} /\left(1-r_{j}\right) .
\end{array}\right\}
$$

where $r_{j}(j=1, \cdots, 6)$ are randomly determined values between 0 and 1 , and see Eq. (15) for the definition of other symbols. Results of natural frequencies of the first 6 modes for wing structures defined by points along the path with $n_{1}=0.0031, n_{2}=0.9999, n_{3}=0.2089, n_{4}=64.7024, n_{5}=0.9067$ and $n_{6}=0.5325$ are shown in Fig. $9(\mathrm{~b})$, where it can be seen that results by the EPA and the ESA agree with each other quite well.

For an arbitrary new design whose plan form is shown in Fig. 10(a), a down-ward (- $z$ direction) point force of $1 \mathrm{lb}$ is applied at the mid-point of the wing tip. Fig. 10(b) shows displacement components along the leading-edge by the EPA and the ESA, compared with FEA using MSC/NASTRAN. Figs. 10(c) and 10(d) show the Von Mises stress distributions at the wing root and the central span-wise line respectively together with the FEA results. Comparison of the natural frequencies of this wing as given by the EPA, the ESA and the FEA is shown in Table 2. Again, it can be seen that the EPA and the ESA results are close, and they all agree quite well with the FEA results. It is noted that a coarser design space III does not worsen the accuracy of the ESA. 
The CPU time savings using the ESA are obvious. For instance, when 6 terms of the Legendre polynomials $(K=6)$ are used, about $85 \%$ less CPU time is spent in evaluating the stiffness and mass matrices compared with the EPA, where matrix evaluation takes about $68 \%$ of the total CPU time when solving the free vibration problem. When $K=8$, about $83 \%$ less CPU time is spent in evaluating the matrices compared with the EPA, where matrix evaluation takes about $65 \%$ of the total CPU time. Generally speaking, the results given by the ESA in design space II and II are as good as those in design space I although the number of variables increases from 4 to 6 .

\section{Conclusion}

An efficient method for the analysis of built-up wing structures using Equivalent Skin Analysis (ESA) has been developed. The CPU time spent on evaluating the matrices, which takes about $63 \sim 68 \%$ the total CPU time in the EPA applying to solve a free vibration problem, is about 5 6 times less when the ESA is used. Three groups of examples with 4 or 6 design variables show that fairly good results can be obtained. This method is very promising to be used at the early stages of wing design.

\section{Acknowledgments}

The authors would like to gratefully acknowledge the support of NASA Langley Research Center for this research through the Grant NAG-1-1884 with Drs. Jerry Housner and John Wang as the Technical Monitors. Thanks are also due to Dr. Eugene Cliff of the AOE Department, Virginia Tech and Mr. Tim Tomlin of the ESM Department, Virginia Tech for their assistance in using Crunch, the College of Engineering's SGI Origin 2000 computer.

\section{$\underline{\text { References }}$}


I. Kapania, R. K. and Liu, Y., "Static and Vibration Analyses of General Wing Structures Using Equivalent Plate Models", to appear, AIAA Journal. Also presented as Paper 2000-1434 at $4 l^{\prime \prime}$ AIAA/ASME/ASCE/AHS/ASC Structures, Structural Dynamics, and Materials Conference \& Exhibit, 3-6 April 2000, Atlanta, Georgia

2. Livne, E., "Equivalent Plate Structural Modeling for Wing Shape Optimization Including Transverse Shear", AIAA Journal, Vol. 32, No. 6, 1994, pp. 1278-1288.

3. Kapania, R. K. and Lovejoy, A. E., "Free Vibration of Thick Generally Laminated Cantilever Quadrilateral Plates", AIAA Journal, Vol. 34, No. 7, 1996, pp. 1474-1480.

4. Cortial, F., "Sensitivity of Aeroelastic Response of Wings Using Equivalent Plate Models", Technical Report, Department of Aerospace and Ocean Engineering, Virginia Tech, 1996.

5. Haykin, S., Neural Networks: A Comprehensive Foundation, Macmillan College Publishing Company, 1994

6. Hajela, P. and Berke, L., "Neurobiological Computational Models in Structural Analysis and Design”, Computers \& Structures, Vol.41, No. 4, 1991, pp. 657-667

7. Abdalla, K. M. and Stavroulakis, G. E., "A backpropagation Neural Network Model for SemiRigid Steel Connections", Microcomputers in Civil Engineering, Vol. 10, 1995, pp. 77-87

8. Vanluchene, R. D. and Sun, R., "Neural Network in Structural Engineering", Microcomputers in Civil Engineering, Vol. 5, 1990, pp. 207-215

9. Gunaratnam, D. J., and Gero, J. S., "Effect of Representation on the Performance of Neural Networks in Structural Engineering Applications", Microcomputers in Civil Engineering, Vol. 9, 1994. pp. $97-108$ 
10. Liu, Y., Kapania, R. K. and VanLandingham, H., "Simulating and Synthesizing Substructures Using Neural Network and Genetic Algorithms", Modeling and Simulation Based Engineering. ed.

S. N. Atluri \& P. E. ODonoghue, Tech Science Press, 1998, Vol. I. pp. 576-581

11. Kapania, R. K. and Liu, Y., "Applications of Artificial Neural Networks in Structural Engineering with Emphasis on Continuum Models", Technical Report, Virginia Polytechnic Institute and State University, June 1998.

12. Kapania, R. K. and Liu, Y., "Efficient Simulation of Wing Modal Response: Application of $2^{\text {nd }}$ Order Shape Sensitivities and Neural Networks", submitted to $8^{\text {th }}$ AIAA/NASAVUSAF/ISSMO Symposium on Multidiscipline Analysis and Optimization, 6-8 September 2000, Long Beach, CA. 13. Karamcheti, K., Principles of ideal-fluid aerodynamics, R. E. Krieger Pub. Co., New York, 1980.

Table 1 Natural frequencies $(\mathrm{rad} / \mathrm{sec})$ of the wing in Fig. 7(a)

\begin{tabular}{|c|c|c|c|c|c|}
\hline Mode No. & 1 & 2 & 3 & 4 & 5 \\
\hline Mode Shape & $1^{\text {st }}$ bending & $2^{\text {nd }}$ bending & $1^{\text {st }}$ torsion & In plane & $2^{\text {nd }}$ torsion \\
\hline EPA & 279.3 & 982.8 & 1057.5 & 1447.4 & 1945.5 \\
\hline ESA & 274.5 & 984.1 & 1045.1 & 1440.3 & 1936.3 \\
\hline FEM & 279.9 & 965.6 & 973.5 & 1454.4 & 1830.8 \\
\hline
\end{tabular}

Table 2 Natural frequencies $(\mathrm{rad} / \mathrm{sec})$ of the wing in Fig. 10(a)

\begin{tabular}{|c|c|c|c|c|c|}
\hline Mode No. & 1 & 2 & 3 & 4 & 5 \\
\hline Mode Shape & $1^{\text {st }}$ bending & $2^{\text {nd }}$ bending & In plane & $1^{\text {st }}$ torsion & $3^{\text {rd }}$ bending \\
\hline EPA & 71.9 & 233.9 & 358.1 & 452.2 & 479.9 \\
\hline ESA & 70.8 & 239.4 & 358.4 & 469.4 & 504.8 \\
\hline FEM & 66.0 & 222.6 & 377.0 & 413.1 & 468.0 \\
\hline
\end{tabular}




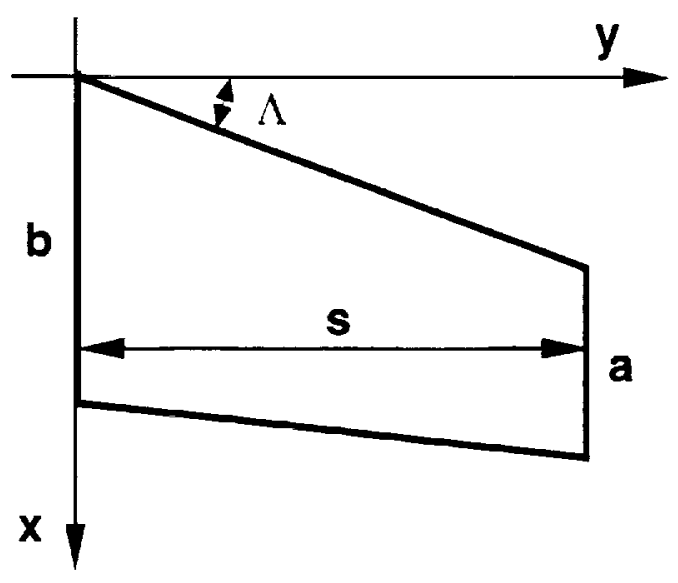

Fig. 1 Plan configuration of a trapezoidal wing: $A=\frac{1}{2} s(a+b), \alpha=s^{2} / A, \tau=a / b$.
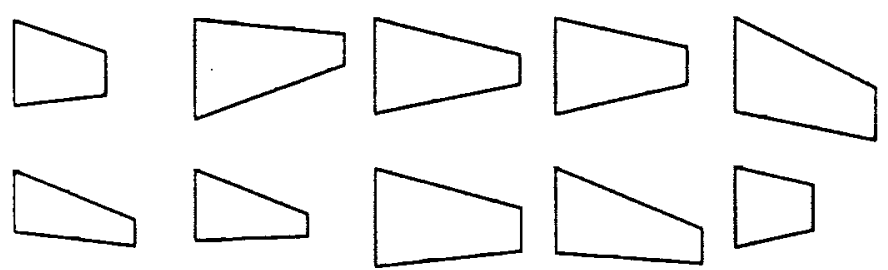<smiles>C1CCC1</smiles>
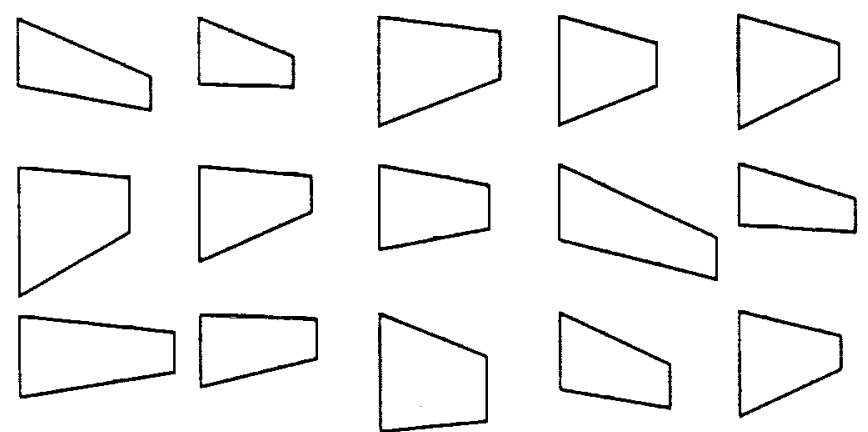

Fig. 2 (a) 25 randomly chosen wing plan forms in design space I 


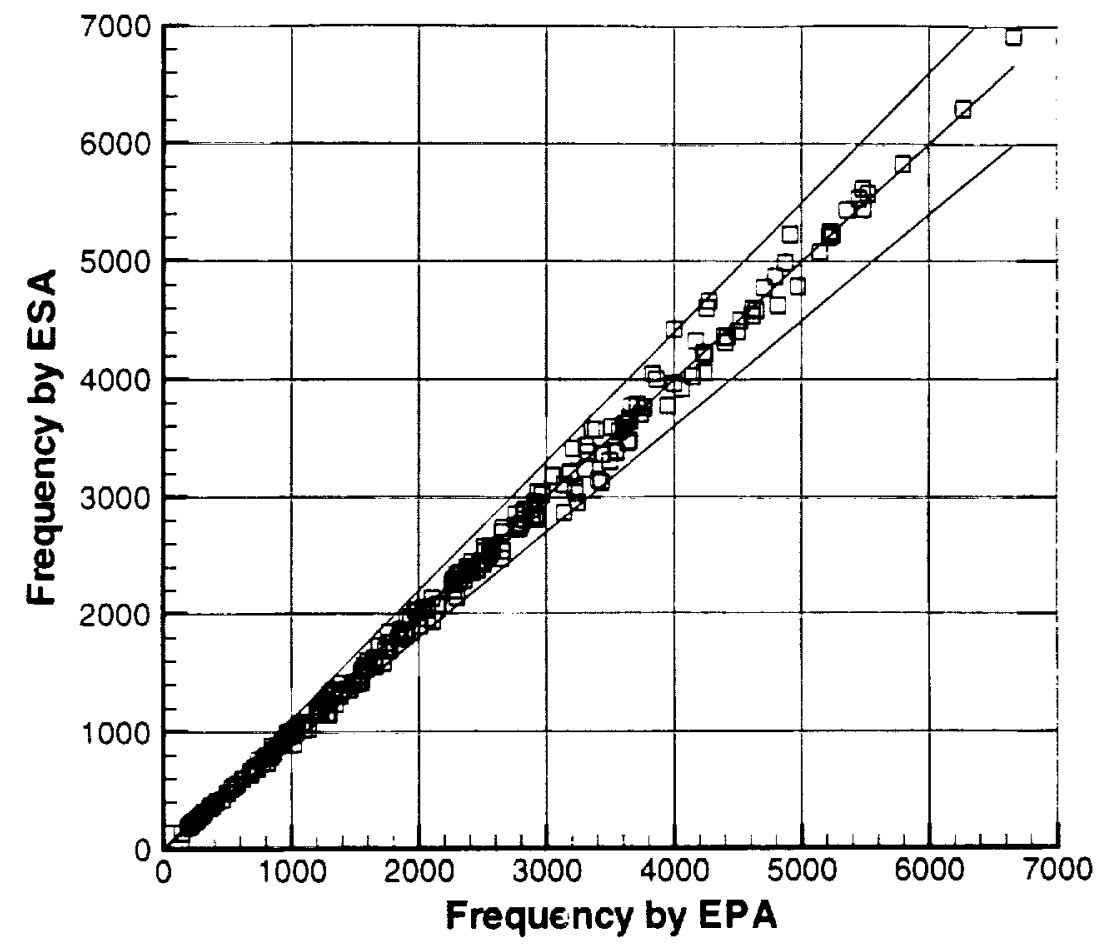

Fig. 2 (b) Comparison of the first 10 frequencies by EPA and ESA

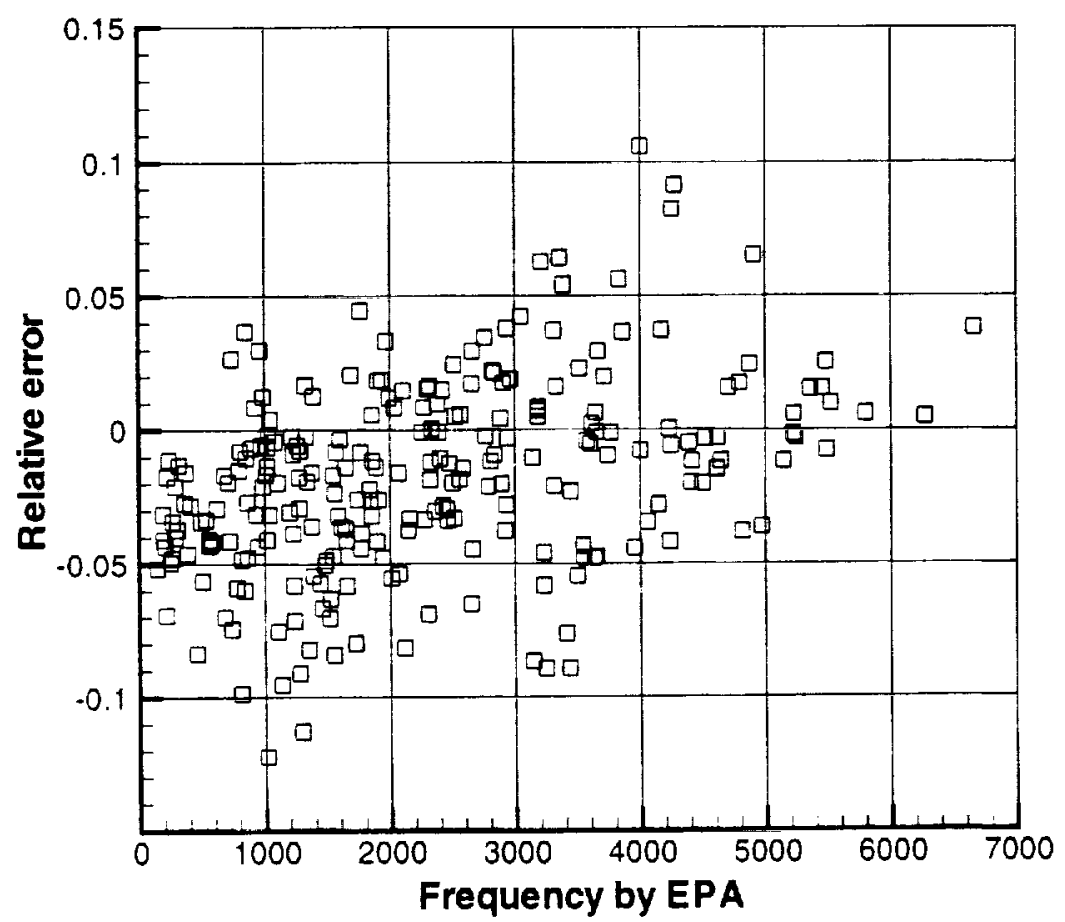

Fig. 2 (c) The relative errors $\left(\left\{f_{E S A}-f_{E P A}\right\} / f_{E P A}\right)$ in (b) 

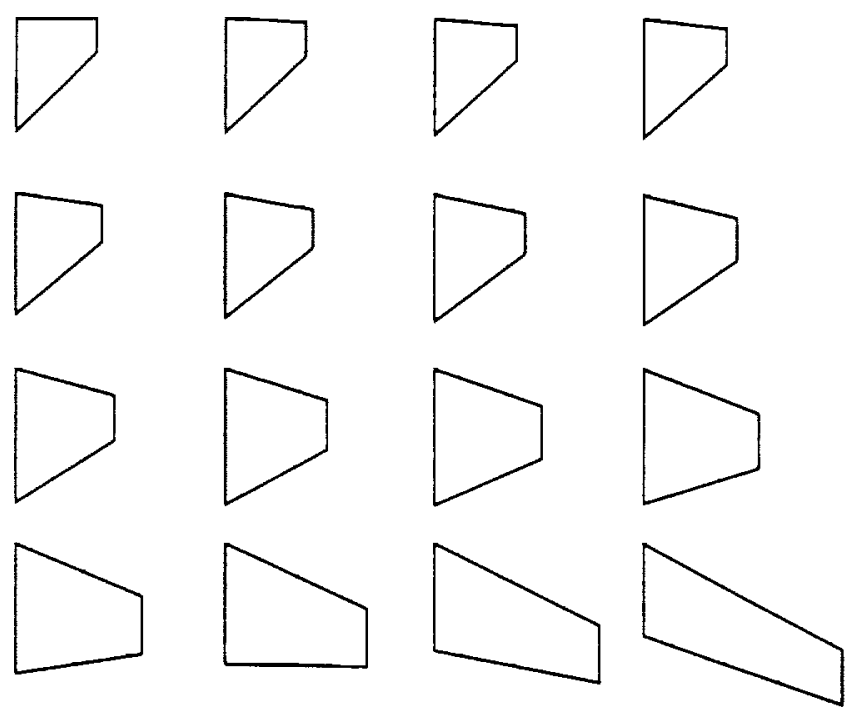

Fig. 3 (a) 16 wing plan forms systematically varying through design space I

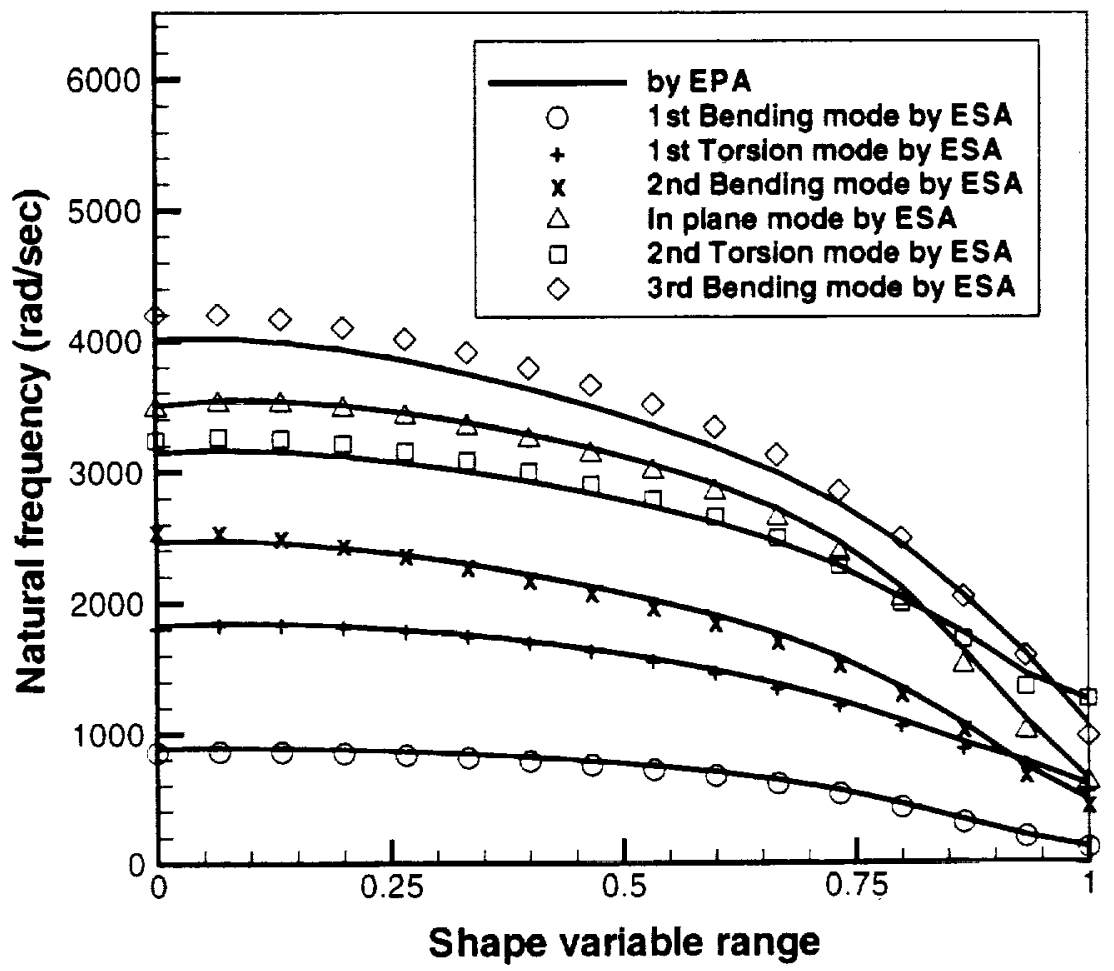

Fig. 3 (b) Comparison of the first 6 frequencies by EPA and ESA 


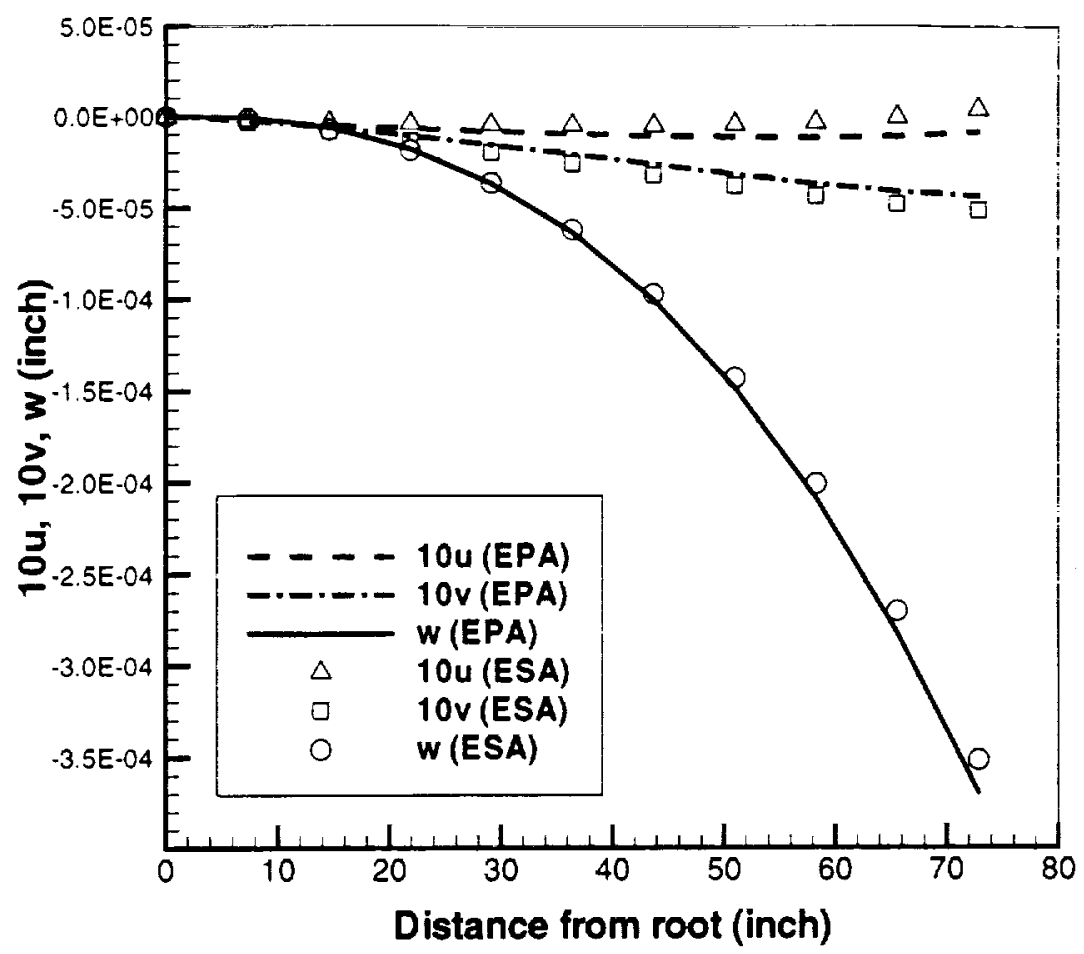

Fig. 4 (a) Comparison of displacements by EPA and ESA under $1 l b$ tip force

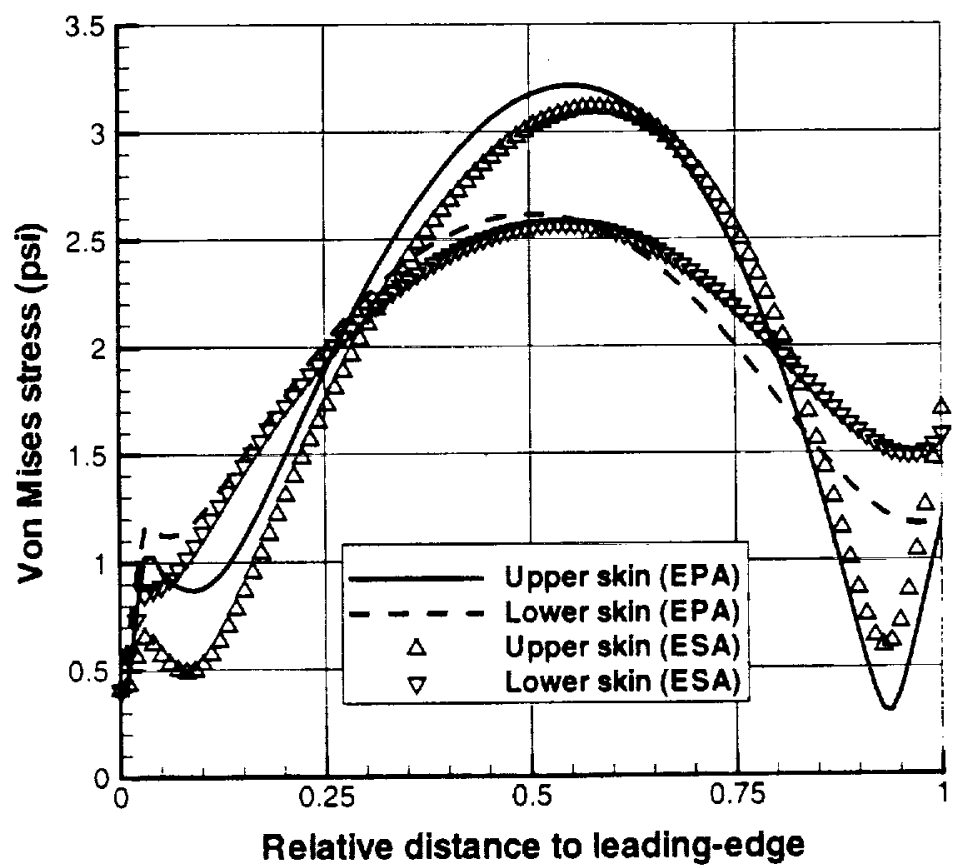

Fig. 4 (b) Comparison of the Von Mises stress at wing root by EPA and ESA under $1 l b$ tip force 


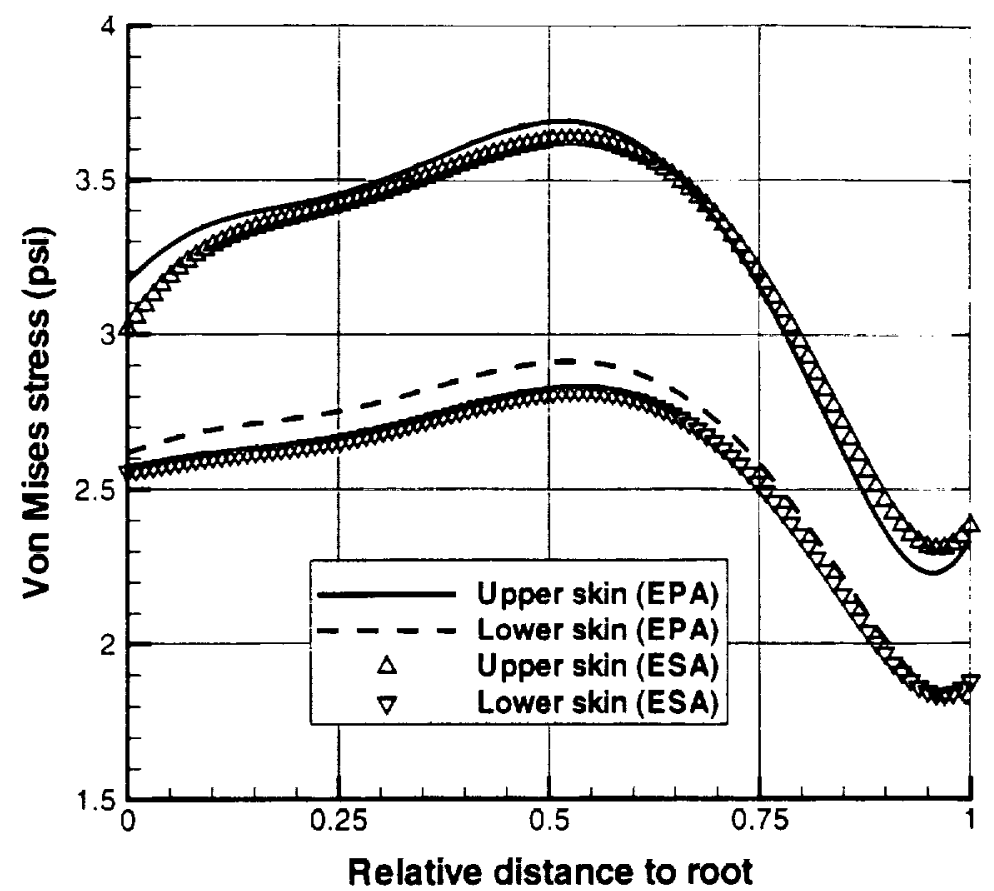

Fig. 4 (c) Comparison of the Von Mises stress along central span-wise line by EPA and ESA under $1 l b$ tip force

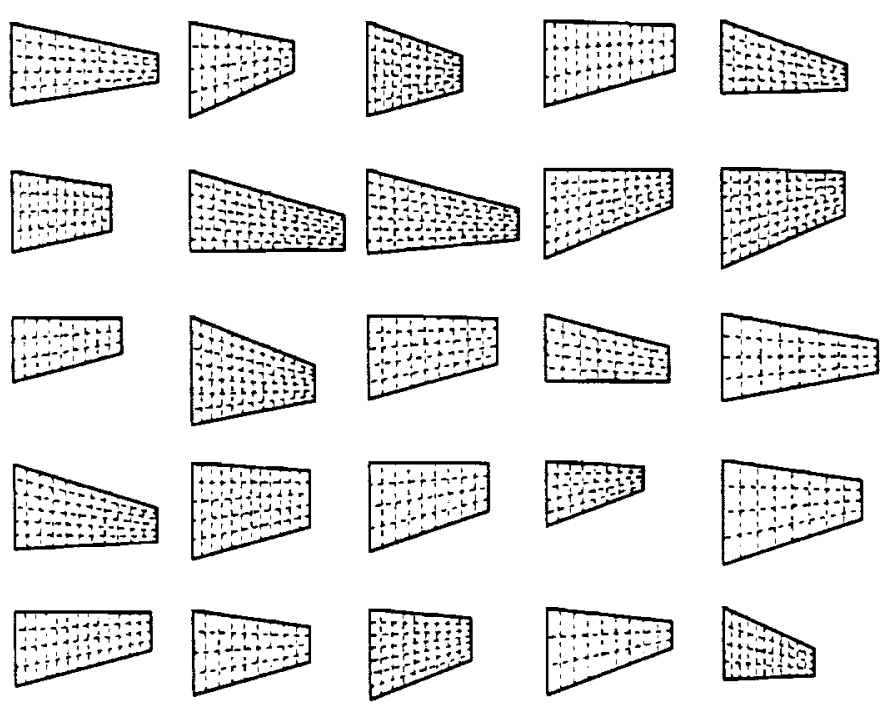

Fig. 5 (a) 25 randomly chosen wing plan forms in design space II 


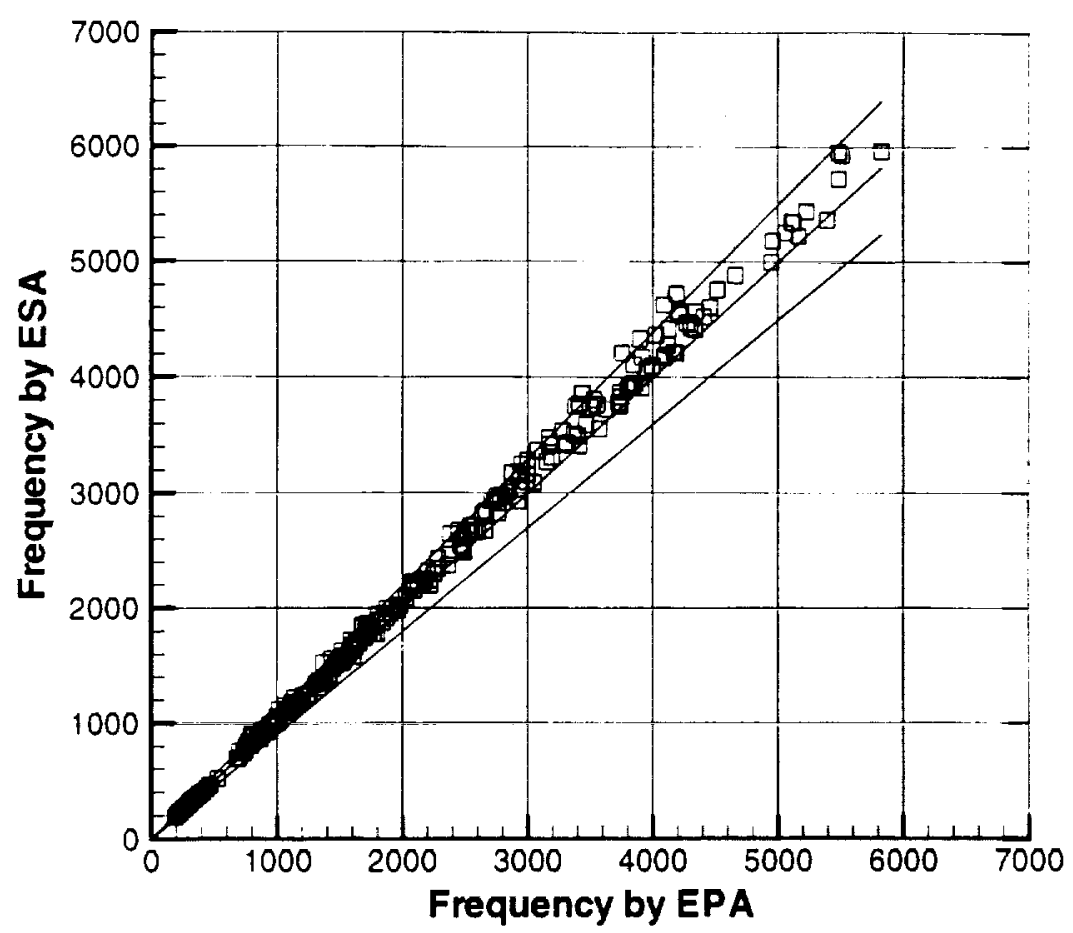

Fig. 5 (b) Comparison of the first 10 frequencies by EPA and ESA

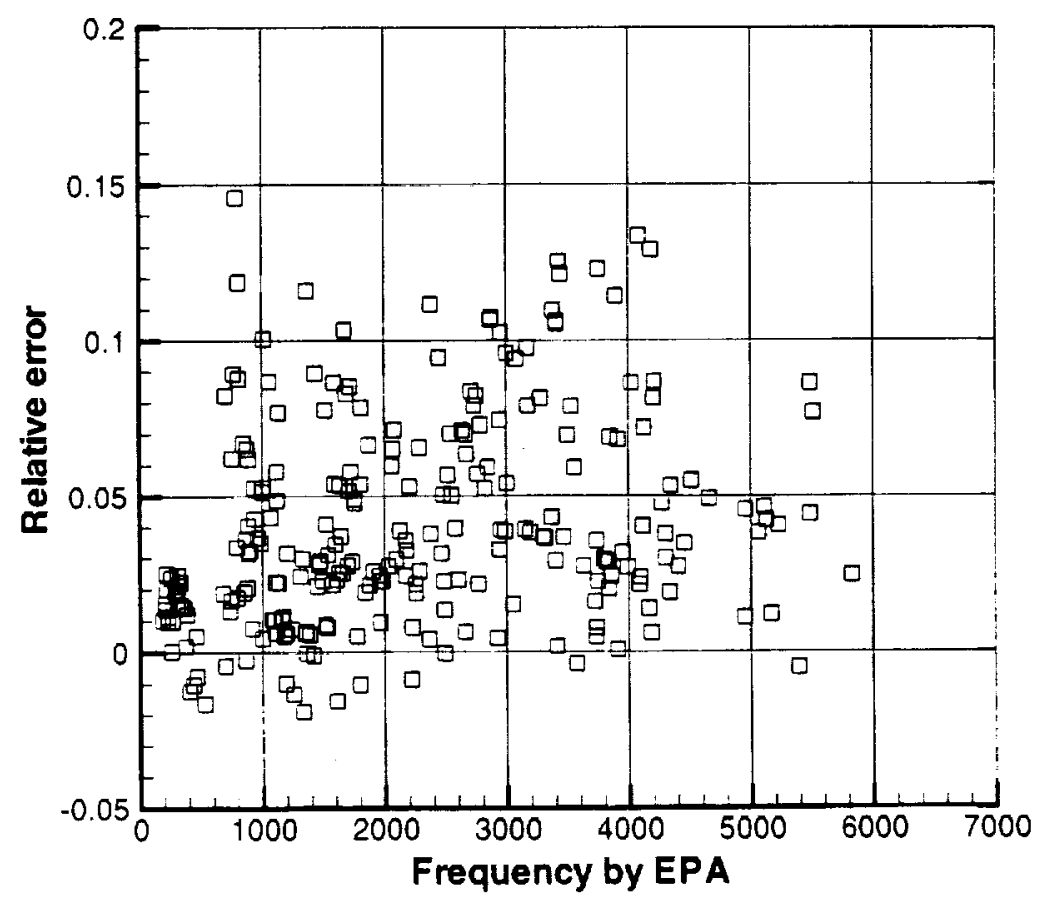

Fig. 5 (c) The relative errors $\left(\left\{f_{E S A}-f_{E P A}\right\} / f_{E P A}\right)$ in (b) 


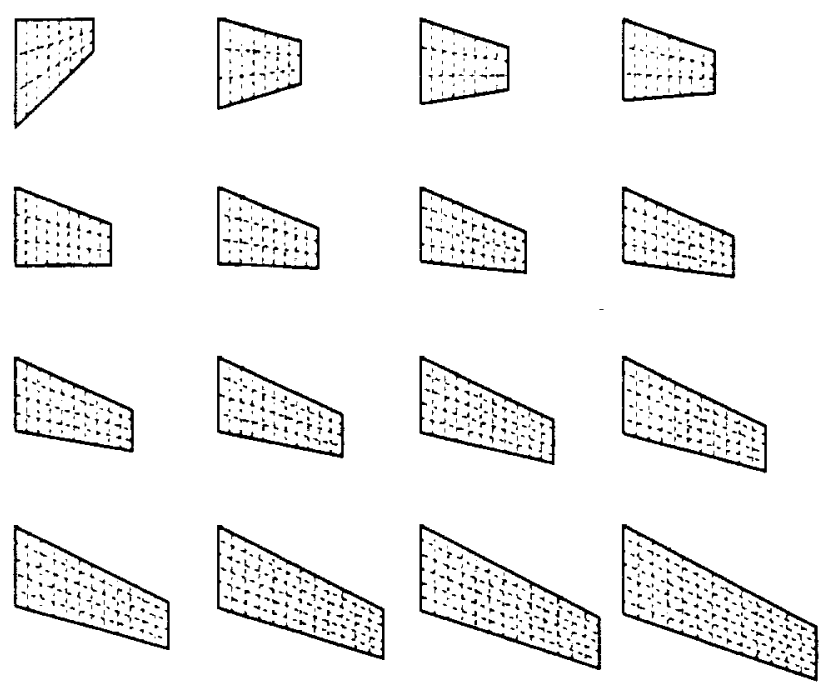

Fig. 6 (a) 16 wing plan forms systematically varying through design space II

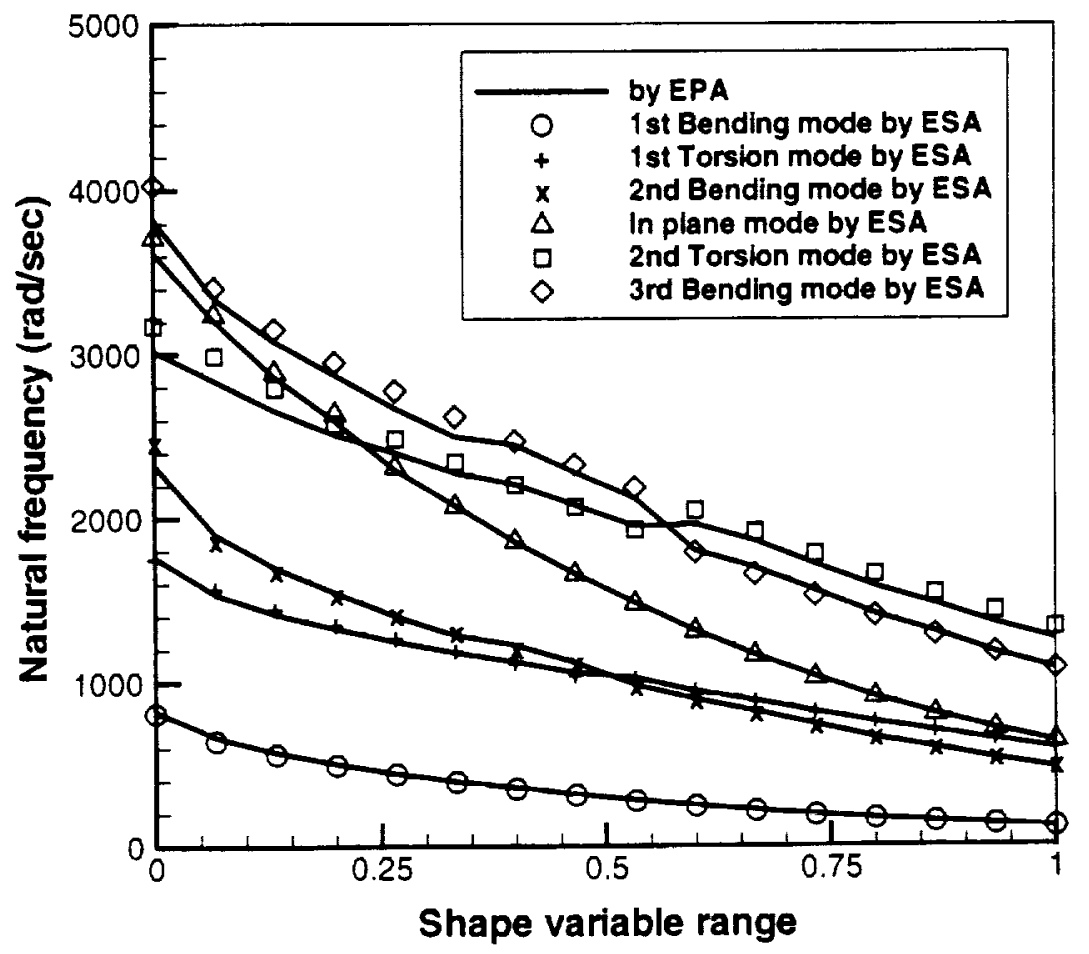

Fig. 6 (b) Comparison of the first 6 frequencies by EPA and ESA 


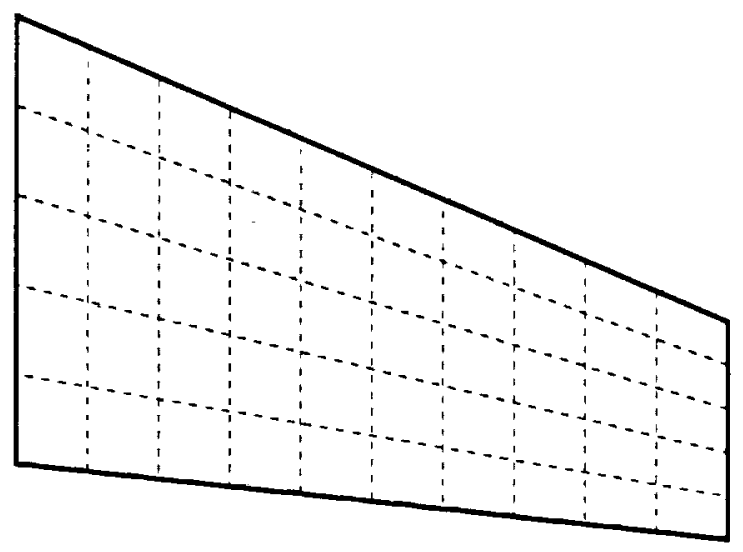

Fig. 7 (a) An arbitrarily chosen wing plan form in design space I \& II

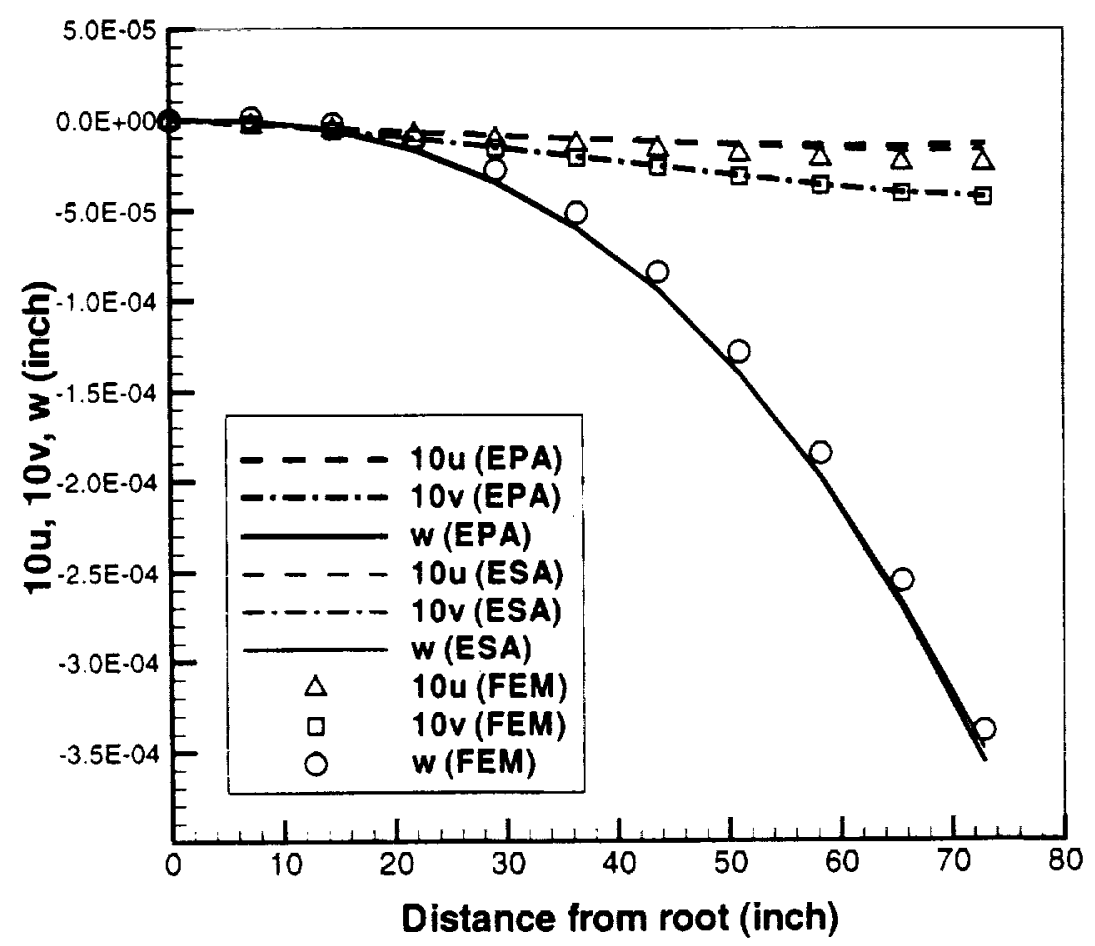

Fig. 7 (b) Comparison of displacements by EPA and ESA under $1 \mathrm{lb}$ tip force 


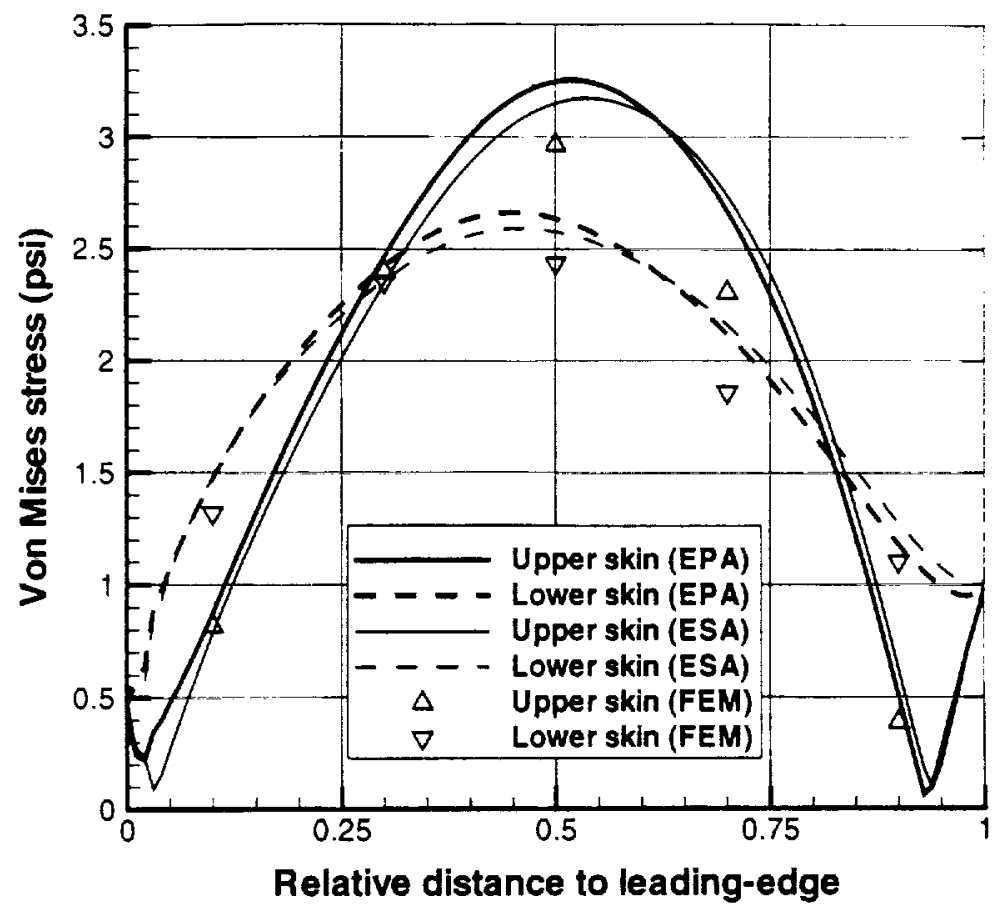

Fig. 7 (c) Comparison of the Von Mises stress at wing root by EPA and ESA under $1 l b$ tip force

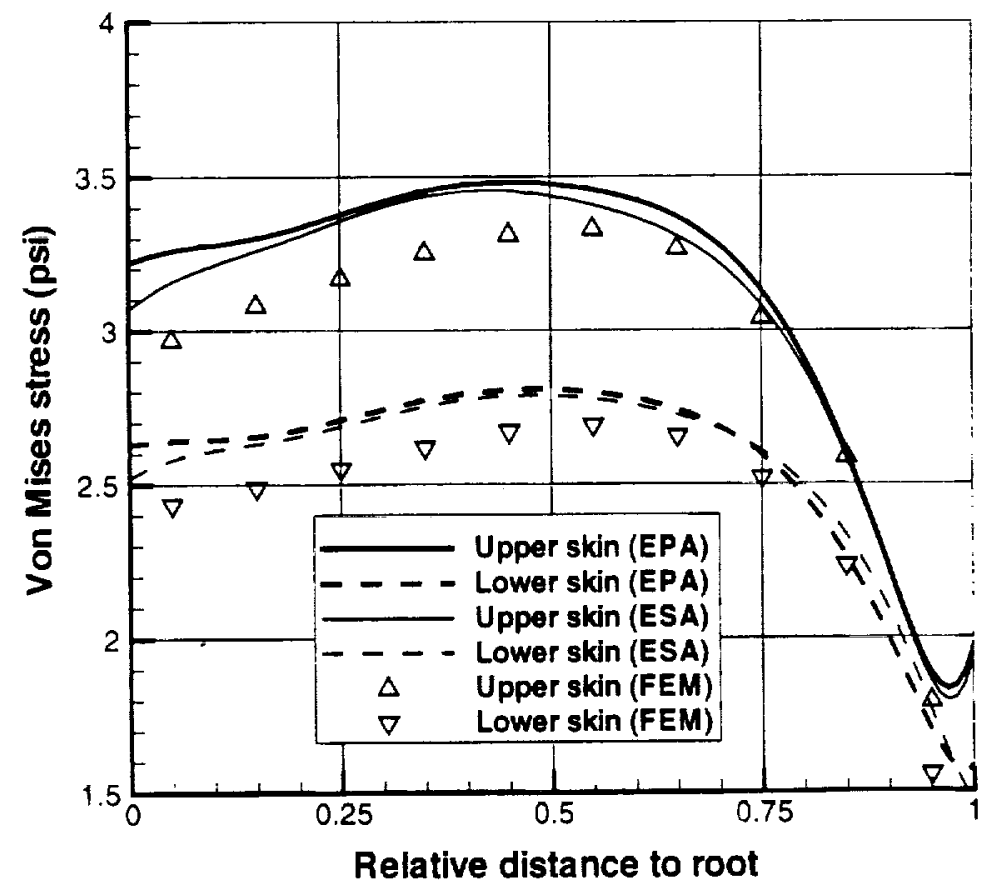

Fig. 7 (d) Comparison of the Von Mises stress along central span-wise line by EPA and ESA under $1 l b$ tip force 

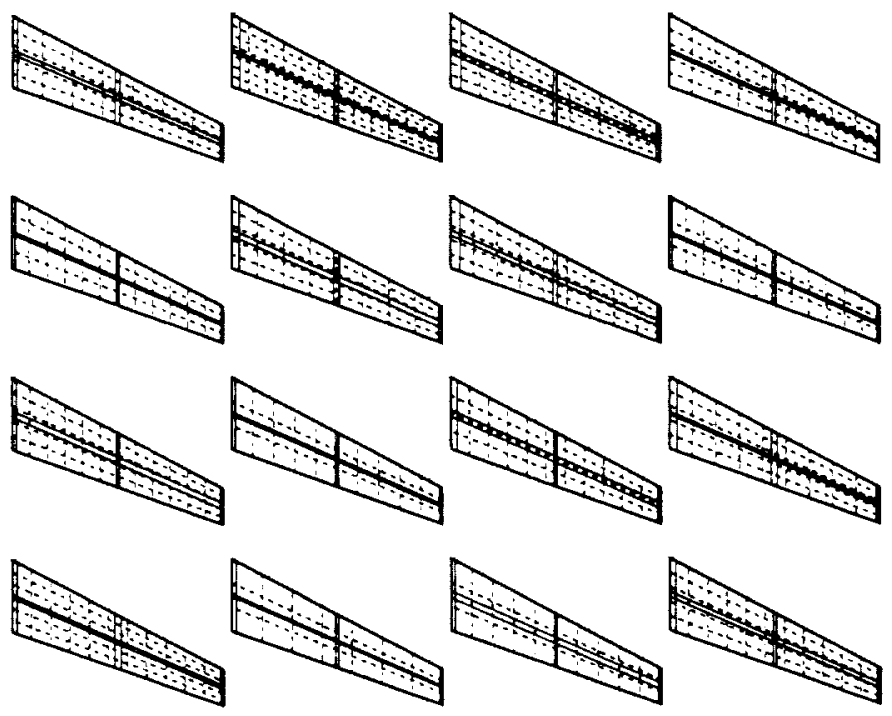

Fig. 8 (a) 16 randomly chosen wing designs in design space III

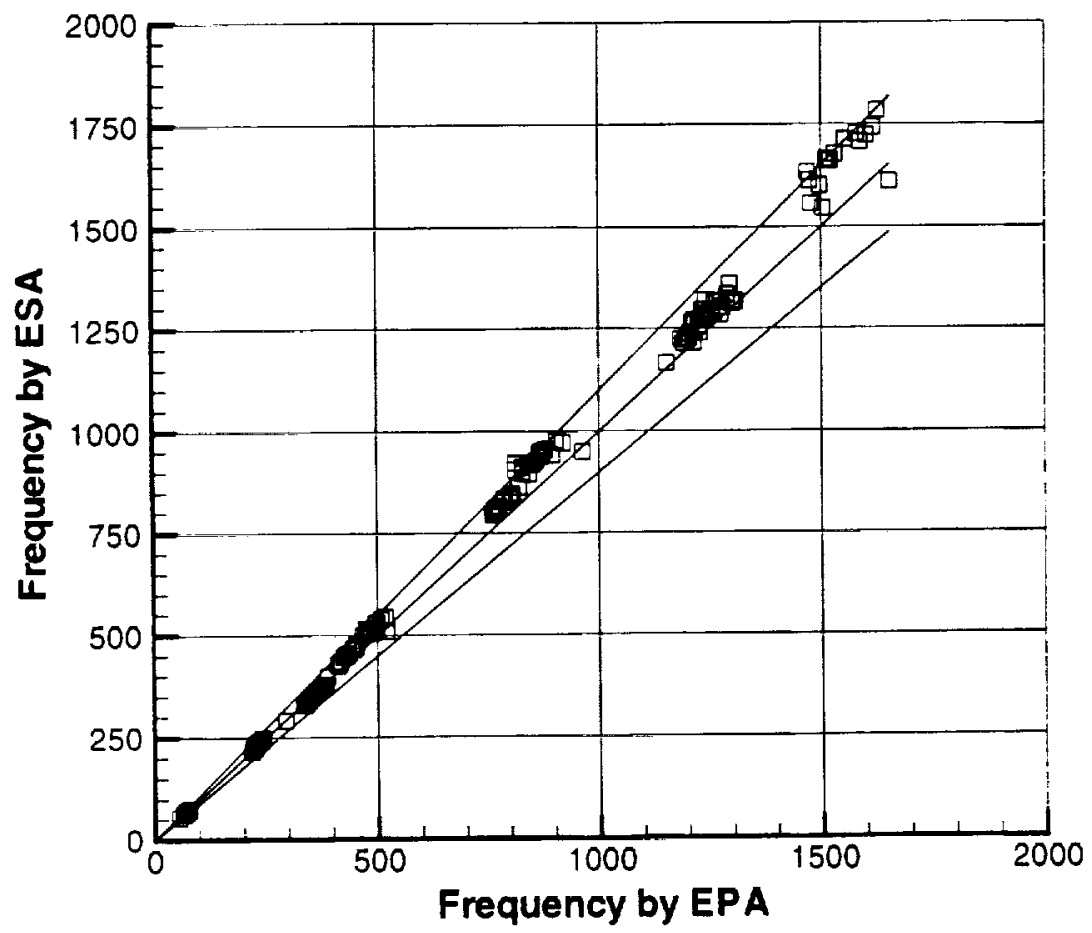

Fig. 8 (b) Comparison of the first 10 frequencies by EPA and ESA 


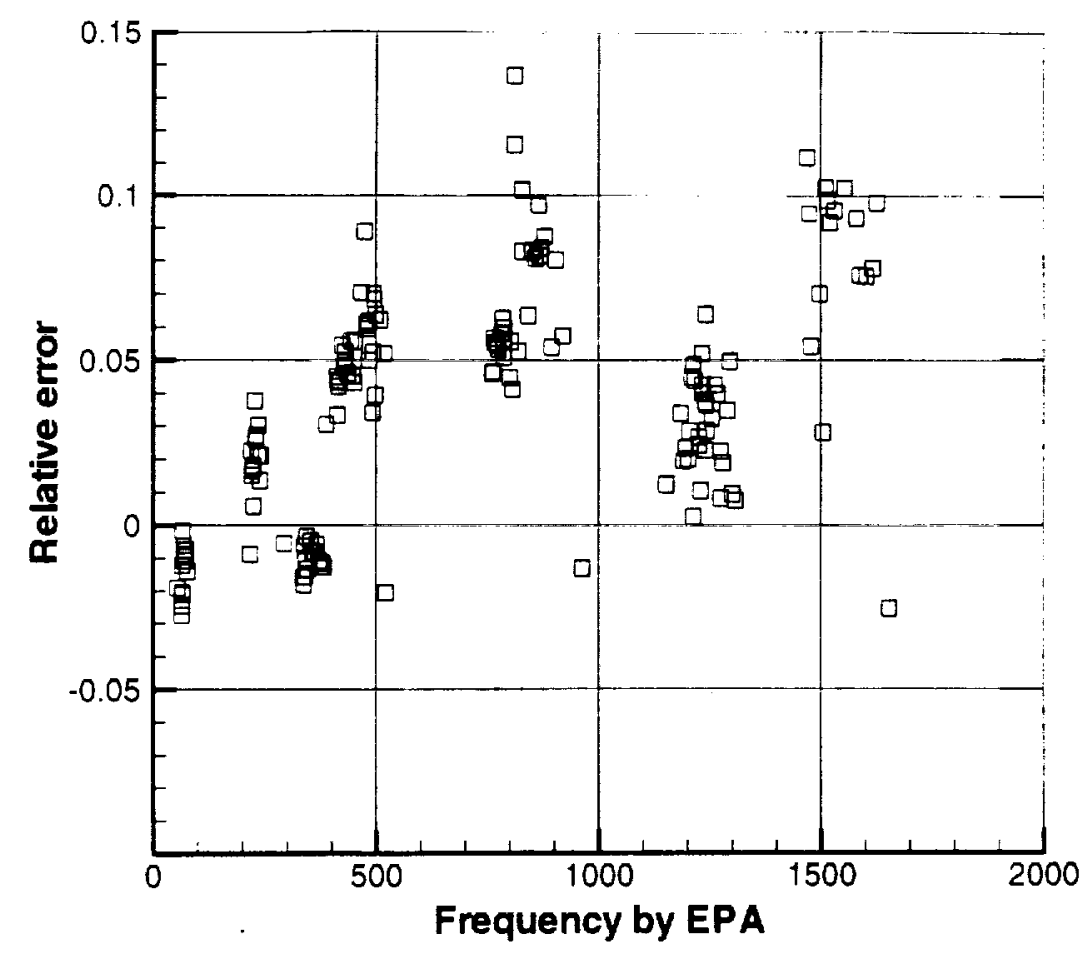

Fig. 8 (c) The relative errors $\left(\left\{f_{E S A}-f_{E P A}\right\} / f_{E P A}\right)$ in (b)
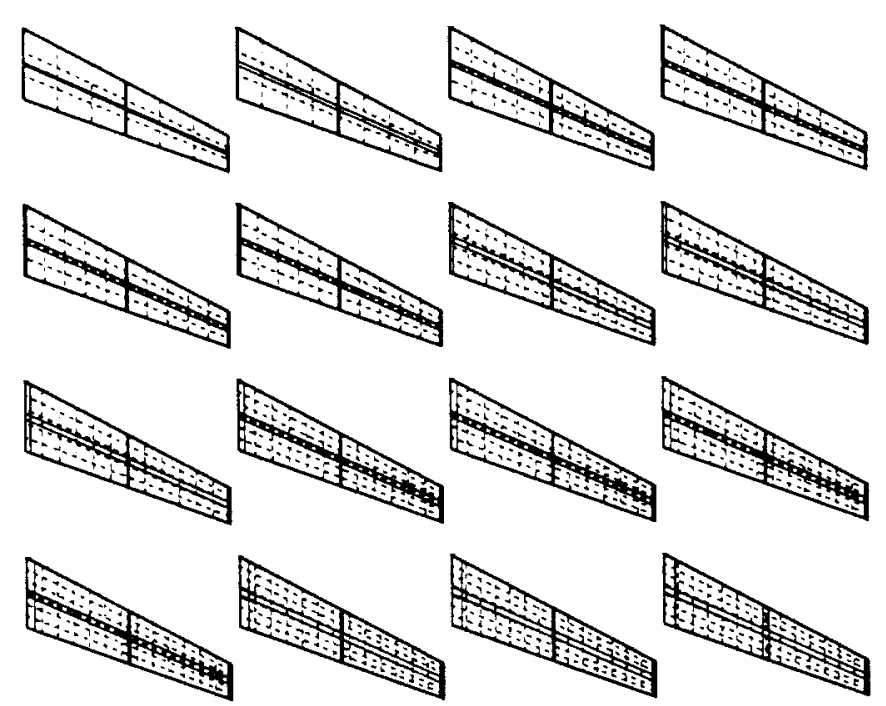

Fig. 9 (a) 16 wing designs systematically varying through design space III 


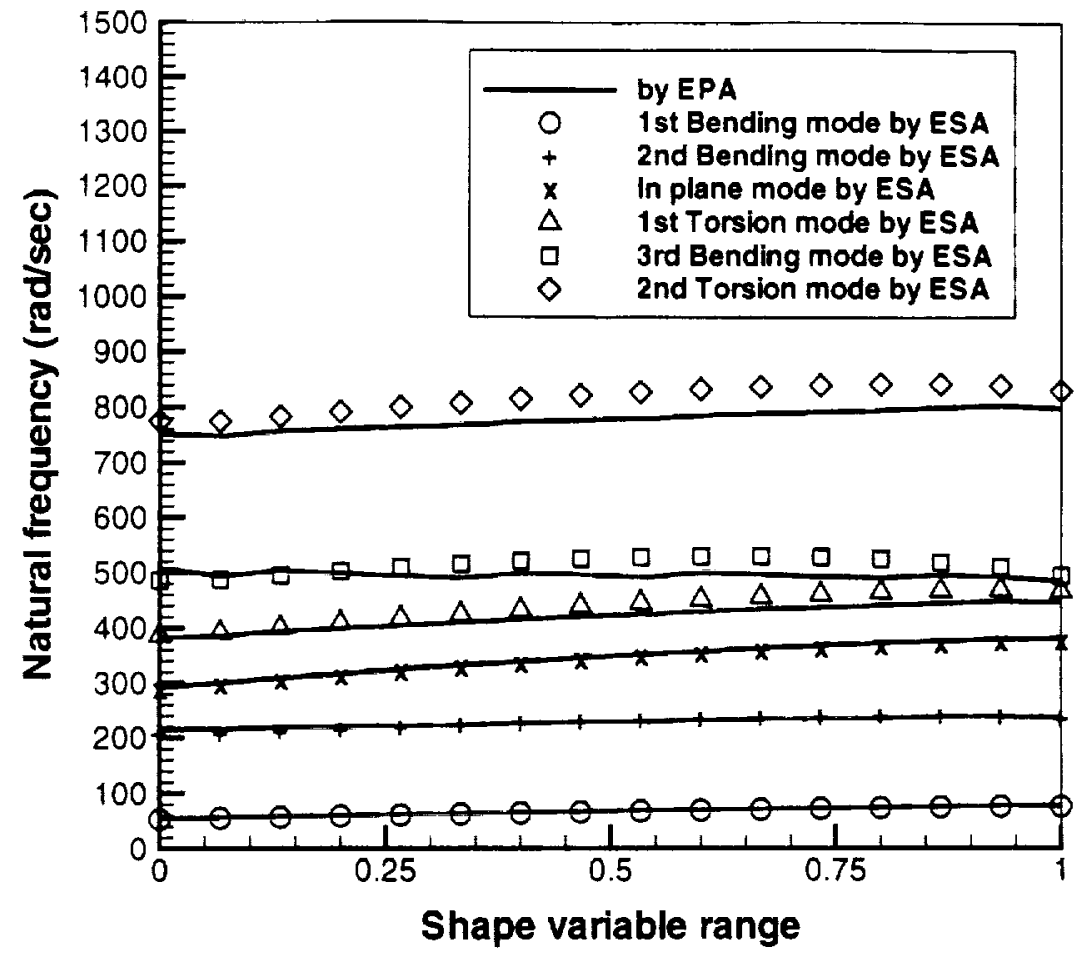

Fig. 9 (b) Comparison of the first 6 frequencies by EPA and ESA

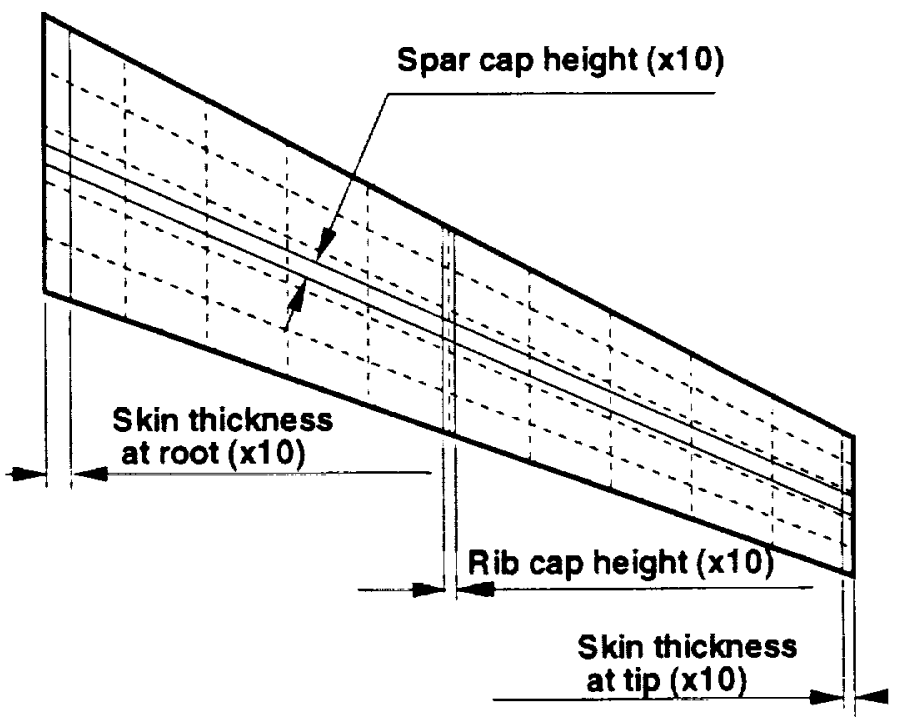

Fig. 10 (a) An arbitrarily chosen wing design in design space III 


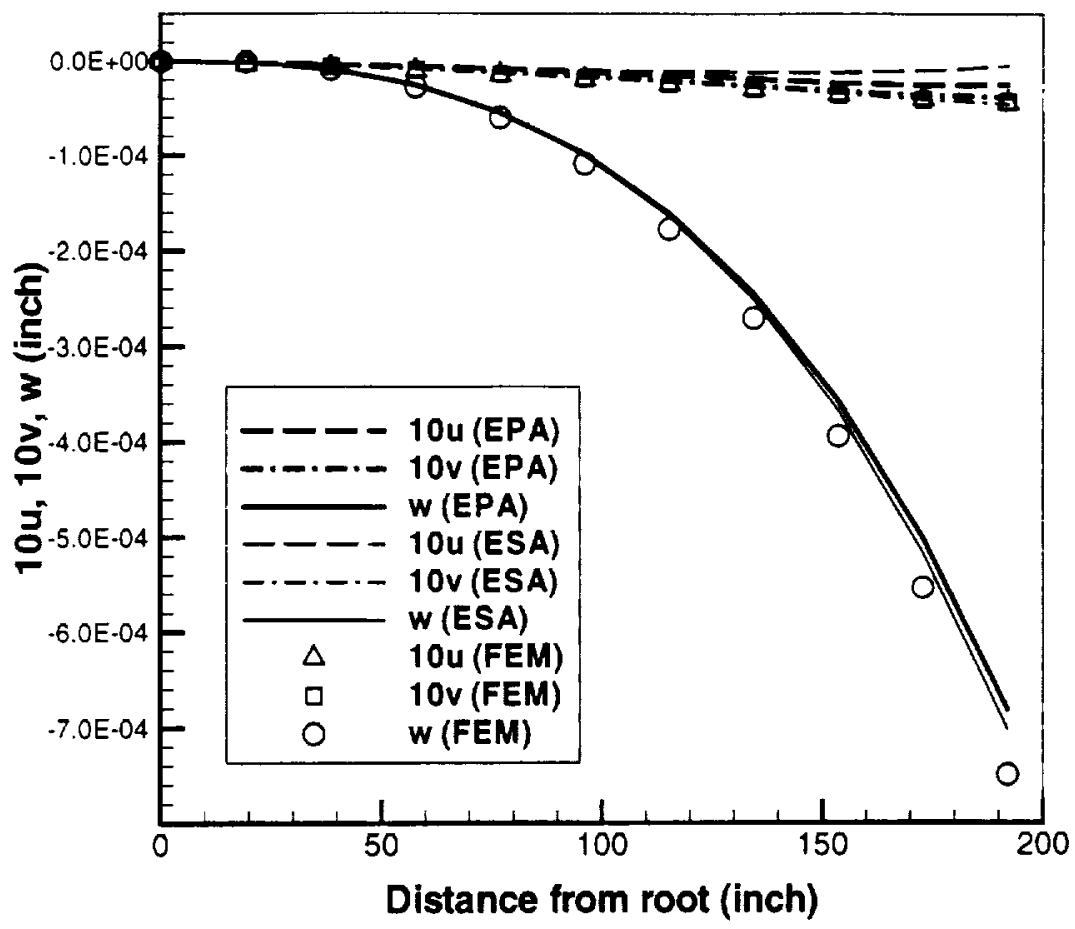

Fig. 10 (b) Comparison of displacements by EPA and ESA under $1 l b$ tip force

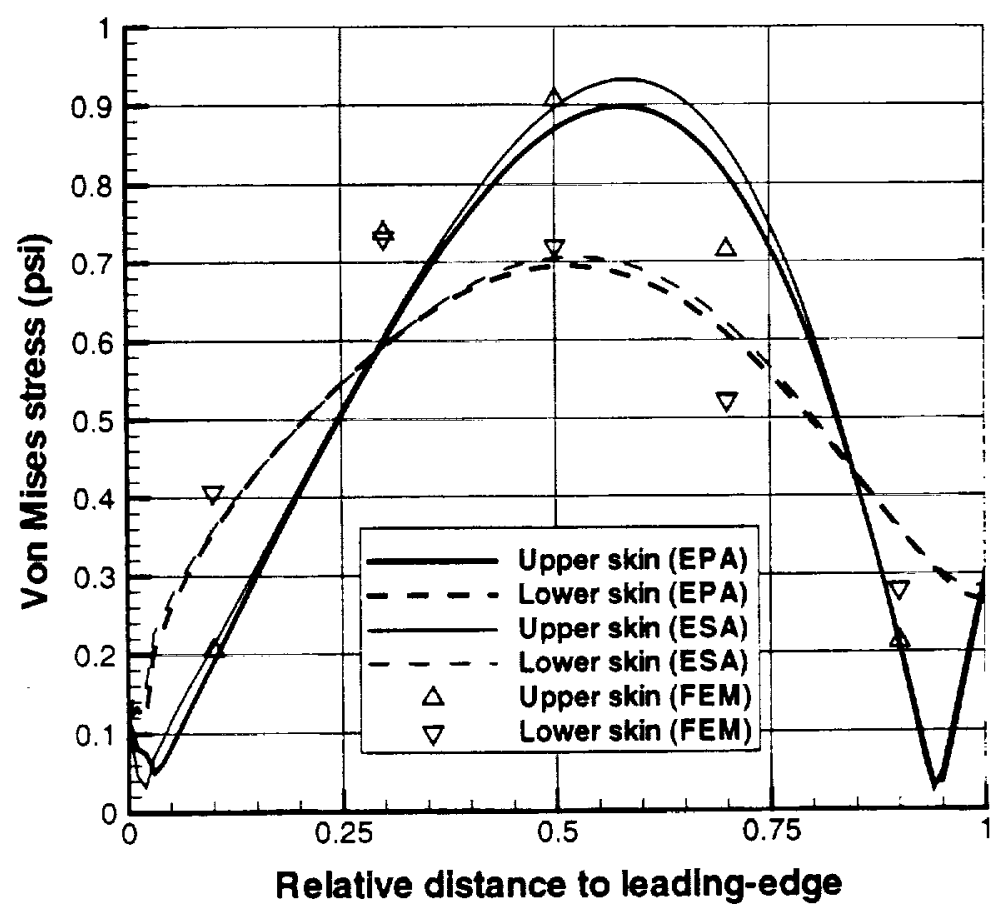

Fig. 10 (c) Comparison of the Von Mises stress at wing root by EPA and ESA under $1 l b$ tip force 


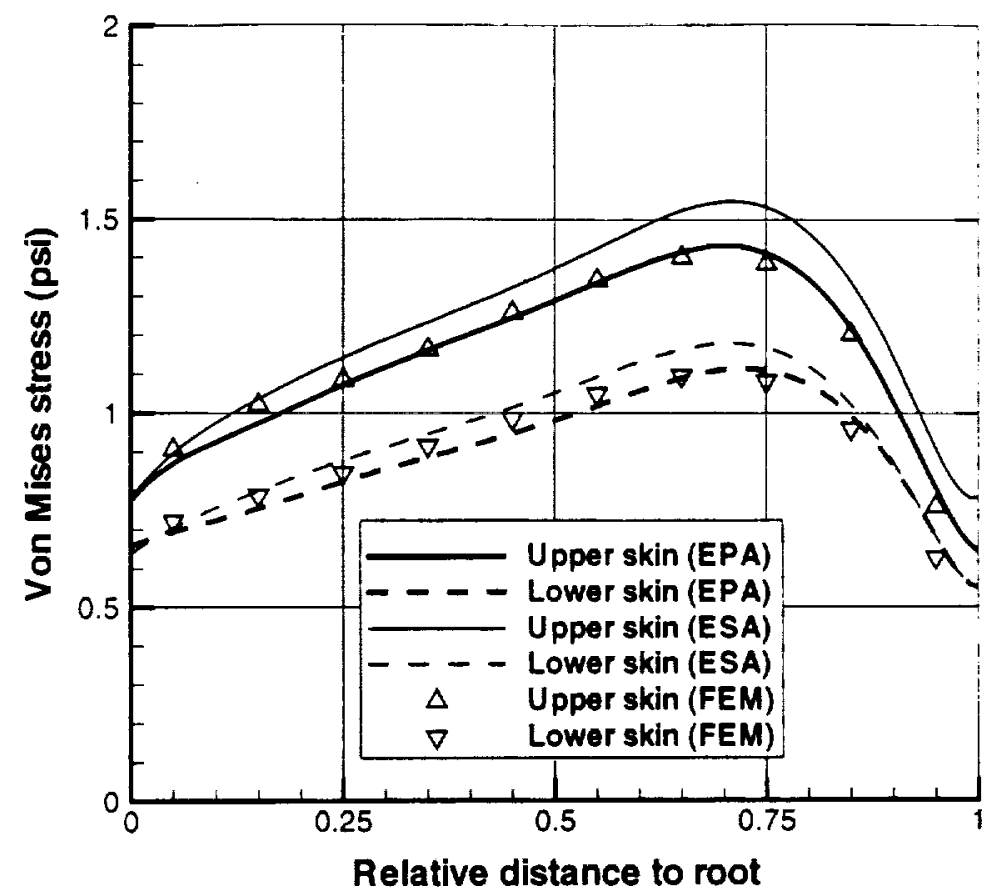

Fig. 10 (d) Comparison of the Von Mises stress along central span-wise line by EPA and ESA under $1 l b$ tip force 